Linköping Studies in Science and Technology

Licentiate Thesis No. 1850

\title{
A Data-centric Internet of Things Framework Based on Public Cloud
}

Yu Liu 


\section{A Data-centric Internet of Things Framework Based on Public Cloud}

\section{Yu Liu}

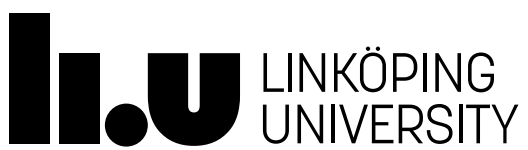

Linköping University

Department of Science and Technology

Division of Physics, Electronics and Mathematics

SE-601 74 Norrköping, Sweden

Norrköping 2019 
This is a Swedish Licentiate's Thesis

Swedish postgraduate education leads to a doctor's degree and/or a licentiate's degree.

\section{Edition 1:1}

(C) Yu Liu, 2019

ISBN 978-91-7519-013-6

ISSN 0280-7971

Published articles have been reprinted with permission from the respective copyright holder.

Typeset using $\mathrm{LT}_{\mathrm{E}} \mathrm{X}$

Printed by LiU-Tryck, Linköping 2019 


\begin{abstract}
The pervasive application of Internet of Things (IoT) has been seen in many aspects in human daily life and industrial production. The concept of IoT originates from traditional machineto-machine (M2M) communications which aimed at solving domain-specific and applicationspecific problems. Today, the rapid progress of communication technologies, the maturation of Internet infrastructures, the continuously reduced cost of sensors, and emergence of more open standards, have witnessed the approaching of the expected IoT era, which envisions full connectivity between the physical world and the digital world via the Internet protocol. The popularity of cloud computing technology has enhanced this IoT transform, benefiting from the superior computing capability and flexible data storage, let alone the security, reliability and scalability advantages.
\end{abstract}

However, there are still a series of obstacles confronted by the industry in deployment of IoT services. First, due to the heterogeneity of hardware devices and application scenarios, the interoperability and compatibility between link-layer protocols, sub-systems and back-end services are significantly challenging. Second, the device management requires a uniform scheme to implement the commissioning, communication, authorization and identity management to guarantee security. Last, the heterogeneity of data format, speed and storage mechanism for different services pose a challenge to further data mining.

This thesis aims to solve these aforementioned challenges by proposing a data-centric IoT framework based on public cloud platforms. It targets at providing a universal architecture to facilitate the deployment of IoT services in massive IoT and broadband IoT categories. The framework involves three representative communication protocols, namely WiFi, Thread and LoRaWAN, to enable support for local, personal, and wide area networks. A security assessment taxonomy for wireless communications in building automation networks is proposed as a tool to evaluate the security performance of adopted protocols, so as to mitigate potential network flaws and guarantee the security. Azure cloud platform is adopted in the framework to provide device management, data processing and storage, visualization, and intelligent services, thanks to the mature cloud infrastructure and the uniform device model and data model. We also exhibit the value of the study by applying the framework into the digitalization procedure of the green plant wall industry. Based on the framework, a remote monitoring and management system for green plant wall is developed as a showcase to validate the feasibility. Furthermore, three specialized visualization methods are proposed and a neuron network-based anomaly detection method is deployed in the project, showing the potential of the framework in terms of data analytics and intelligence. 



\section{Acknowledgments}

First of all, I would like to express my gratitude and respect to my supervisor Professor Shaofang Gong, Docent Magnus Karlsson and Doctor Zhibo Pang, for their guidance and support to my research and to my life. Two years ago, Shaofang provided me this great opportunity to conduct research in a promising topic in $\mathrm{LiU}$ with wonderful platform and environment. Magnus offered me countless help in building up the research platform and shared with me his valuable input. Zhibo always gives me his wise advices about research and career from a high point, which motivated me a lot.

I also would like to thank my colleagues, Adriana Serban, Gustav Knutsson, Qin-Zhong Ye, and Robert Malmqvist, in the Communication Electronic research group, for their valuable input to my study and research.

Moreover, I would like to show my appreciation to all the colleagues in ITN and friends in my life.

Last but not least, I want to thank my family, my parents and my wife. Without their firm support to my life and to my spirit, I could not have gone so far.

Yu Liu,

Norrköping, July 2019 



\section{Publication List}

\section{Papers included in this thesis:}

Paper I: Y. Liu, K. Akram Hassan, M. Karlsson, Z. Pang and S. Gong, "A Data-Centric Internet of Things Framework Based on Azure Cloud," in IEEE Access, vol. 7, pp. 53839-53858, 2019.

Paper II: Y. Liu, Z. Pang, G. Dán, D. Lan and S. Gong, "A Taxonomy for the Security Assessment of IP-Based Building Automation Systems: The Case of Thread," in IEEE Transactions on Industrial Informatics, vol. 14, no. 9, pp. 4113-4123, Sept. 2018.

Paper III: Y. Liu, K. Akram Hassan, M. Karlsson, O. Weister and S. Gong, "Active Plant Wall for Green Indoor Climate Based on Cloud and Internet of Things," in IEEE Access, vol. 6, pp. 33631-33644, 2018.

Paper IV: K. Akram Hassan,Y. Liu, L. Besançon, J. Johansson, and N. Rönnberg, "A Study on Visual Representations for Active PlantWall Data Analysis," in Data 4.2, 2019.

Paper V: Y. Liu, M. Karlsson, Z. Pang and S. Gong, "Towards Predictive Maintenance of Indoor Climate with A Vertical Plant Wall and Neural Networks," Manuscript, 2019.

\section{Papers not included in this thesis:}

Paper VI: E. Azoidou, Z. Pang, Y. Liu, D. Lan, G. Bag and S. Gong, "Battery Lifetime Modeling and Validation of Wireless Building Automation Devices in Thread," in IEEE Transactions on Industrial Informatics, vol. 14, no. 7, pp. 2869-2880, July 2018.

Paper VII: D. Lan, Z. Pang, C. Fischione, Y. Liu, A. Taherkordi and F. Eliassen, "Latency Analysis of Wireless Networks for Proximity Services in Smart Home and Building Automation: The Case of Thread," in IEEE Access, vol. 7, pp. 4856-4867, 2019. 


\section{Contents}

$\begin{array}{ll}\text { Abstract } & \text { iii }\end{array}$

$\begin{array}{ll}\text { Acknowledgments } & \text { v }\end{array}$

Publication List vii

Contents viii

1 Introduction 1

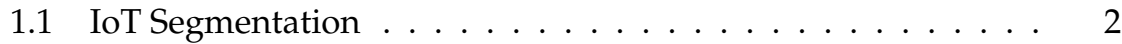

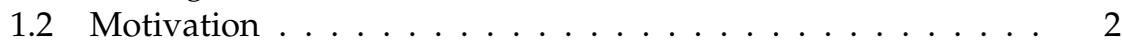

1.3 Aim and Contributions . . . . . . . . . . . . . . . . 4

1.4 Thesis Outline . . . . . . . . . . . . . . . 5

2 Data-centric IoT Framework $\quad 7$

2.1 Background .................. 7

2.2 Framework Overview ................. 9

2.3 Azure Cloud Infrastructure . . . . . . . . . . . . . . . . 10

2.4 Local Infrastructure . . . . . . . . . . . . . . . . . . . . . 13

2.5 Device Model . . . . . . . . . . . . . . . . . . . . . 17

2.6 Data Model . . . . . . . . . . . . . . . . 18

3 Security of The Framework $\quad 21$

3.1 Security Relevant Characteristics . . . . . . . . . . . . . . 21

3.2 Taxonomy for Security Assessment . . . . . . . . . . . . 22

3.3 Security in Thread . . . . . . . . . . . . . . . . . . 24

3.4 Security in WiFi . . . . . . . . . . . . . . . . . . . . 24

3.5 Security in LoRaWAN . . . . . . . . . . . . . . . . . . . . 25

3.6 Cloud Security . . . . . . . . . . . . . . . . . . . . . 25

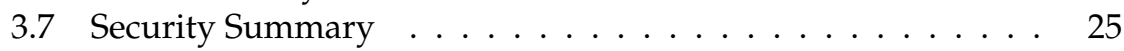

4 Application: Cloud and IoT-powered Green Plant Wall Industry 27

4.1 Green Plant Wall Industry . . . . . . . . . . . . . . . . . . . . 27

4.2 Solution Overview . . . . . . . . . . . . . . 28 
4.3 System Implementation $\ldots \ldots \ldots$

4.4 Visualization . . . . . . . . . . . . . . . . . 32

4.5 Intelligence . . . . . . . . . . . . . . . . . 33

5 Summary of publications 37

6 Conclusion and Future Work 39

$\begin{array}{ll}\text { Bibliography } & 41\end{array}$

7 Paper I 47

7.1 Introduction . . . . . . . . . . . . . . . . . . 48

7.2 Related Work . . . . . . . . . . . . . . . . . . . 50

7.3 Data-centric IoT framework . . . . . . . . . . . . . . . 54

7.4 Implementation . . . . . . . . . . . . . . . . . 66

7.5 Results . . . . . . . . . . . . . . . . . . . . . . 75

7.6 Conclusion . . . . . . . . . . . . . . . . . . 77

7.7 Acknowledgement . . . . . . . . . . . . . . . . . . 79

References . . . . . . . . . . . . . . . . . . 79

8 Paper II $\quad 85$

8.1 Introduction . . . . . . . . . . . . . . . 85

8.2 Related Work . . . . . . . . . . . . . . . . . . . 86

8.3 BAS Security Taxonomy . . . . . . . . . . . . . . . . . 88

8.4 Security in Thread . . . . . . . . . . . . . . . . . . . . . 91

8.5 Validation and Countermeasures . . . . . . . . . . . . . 98

8.6 Conclusion and Future Work . . . . . . . . . . . . . . . . 101

8.7 Acknowledgment . . . . . . . . . . . . . . . . . . 102

References . . . . . . . . . . . . . . . . 102

9 Paper III 109

9.1 Introduction . . . . . . . . . . . . . . . . . . . . . . . . . 109

9.2 Related Work . . . . . . . . . . . . . . . . . . . . . . . 111

9.3 Public Cloud Platform . . . . . . . . . . . . . . . . . . . . . . 114

9.4 System Overview . . . . . . . . . . . . . . . . . . . . . . 115

9.5 System Design and Implementation . . . . . . . . . . . . . 116

9.6 Results . . . . . . . . . . . . . . . . . . . . . . . . 126

9.7 Concluding Remarks . . . . . . . . . . . . . . . . . . . . 128

References . . . . . . . . . . . . . . . . . . . . . . . . . . 129

10 Paper IV 135

10.1 Introduction . . . . . . . . . . . . . . . . . . . 136

10.2 System Setup . . . . . . . . . . . . . . . . . . . 137

10.3 Visual Representations . . . . . . . . . . . . . . . . . . . . 138

10.4 Method . . . . . . . . . . . . . . . . . . . . . . . 141 
10.5 Results . . . . . . . . . . . . . . . . . . . . . 144

10.6 Discussion . . . . . . . . . . . . . . . . 150

10.7 Limitations and Future Work . . . . . . . . . . . . . 151

10.8 Conclusion . . . . . . . . . . . . . . . . . . . 153

10.9 Acknowledgements . . . . . . . . . . . . . . . . . . . . . . . 154

References . . . . . . . . . . . . . . . . . . 154

11 Paper V 163

11.1 Introduction . . . . . . . . . . . . . . . . 163

11.2 Related Work . . . . . . . . . . . . . . . . . . . . . . 166

11.3 Experiment Design . . . . . . . . . . . . . . . . 167

11.4 Experimental Results and Discussion . . . . . . . . . . . 168

11.5 Adoption of the Results . . . . . . . . . . . . . . . . 178

11.6 Conclusion . . . . . . . . . . . . . . . . . . . . 178

References . . . . . . . . . . . . . . . . . . . . 179 


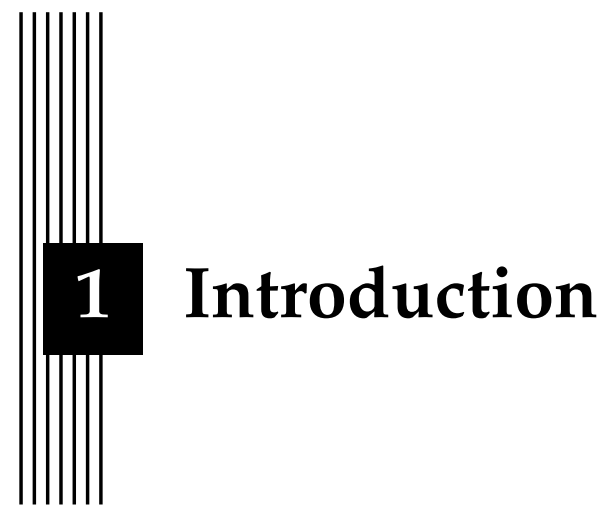

The term Internet of Things (IoT) has been proposed to reflect the connectivity between physical and digital worlds [1]. IoT envisions a society where all the real world assets are highly connected with the Internet infrastructure which involve anything, anytime, anywhere, any service and any media. For years, this vision has gradually shown its pervasive influences on many domains ranging from human daily life to industrial production, indicating a digitization revolution is taking place in all the aspects of the society.

The concept of IoT stems from traditional Machine-to-Machine (M2M) communication technologies which emerges since the 1990s. Different from the M2M paradigm which emphasizes problem-driven solutions to communicate devices of the same type and specific applications, IoT depicts a broader picture of the future communications among devices, services and enterprise processes, which is driven by the increasing need for acquisition of information and innovation in modern society. The transform from the problem-driven M2M communication towards the information and innovation-driven IoT paradigm has been largely accelerated by the improved network capabilities, continuously reduced cost, maturation of the Internet infrastructures, and the open standards [2]. Meanwhile, the emergence of the cloud computing to a large extent reinforces this migration trend towards the IoT era by providing centralized data acquisition and storage, and nearly unlimited processing capabilities, which has become a key enabler of the IoT revolution . 


\subsection{IoT Segmentation}

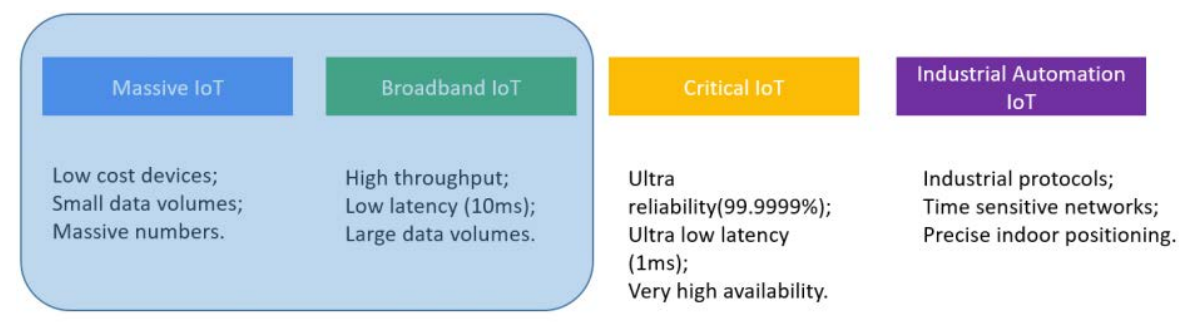

Figure 1.1: IoT Segmentation.

As per Ericsson Research[3], IoT applications are categorized into four segments, namely massive IoT, broadband IoT, critical IoT and industrial automation IoT (see Figure 1.1), according to distinct performance requirements in terms of latency, data rate, data volume, reliability, and availability, etc. Massive IoT targets at low-complexity and low-cost devices that are deployed in a broad range with extended coverage and reliable lifespan, such as sensors, meters, wearables and trackers. Broadband IoT is dedicated to satisfying high throughput and low latency in large data volume transmission. Due to the non-stringent performance requirements, these aforementioned two categories of IoT have been found extensive applications in consumer IoT scenarios, where the services are prone to be human-centered, and Ad-hoc and mobile network connectivity are dominant [4]. As opposed to consumer IoT area, critical IoT and Industrial automation IoT (IIoT) pose much stringent requirements on network performance, which demands ultra-low latency and ultra-high reliability and availability.

A series of typical IoT applications and relevant performance requirements in the smart city paradigm are identified in [5] and [6], which reveals that the majority of the smart city services such as monitoring, metering, automatic vending, building automation, fleet management, and smart parking are characterized with low traffic rate (from one packet per 30 seconds to 12 hours) and tolerable delay (from 10 seconds to 30 minutes). In light of this, an IoT framework that is adapted to accelerating the development of massive IoT and broadband IoT use cases is highly demanded by the industry.

\subsection{Motivation}

\section{Challenges}

The essential goal of IoT is data and the intelligence that are extracted from the data. To transparently and seamlessly incorporate a variety of end devices and systems, while providing digital services based on accumulated 


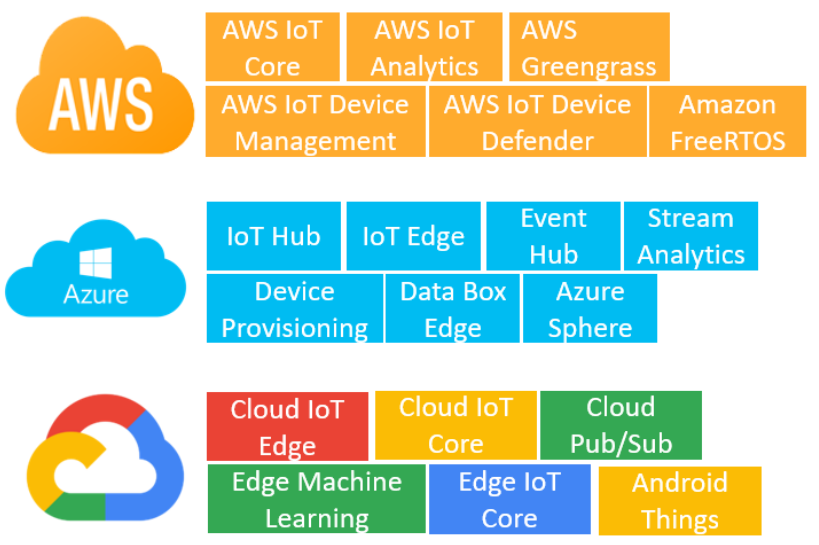

Figure 1.2: IoT infrastructures of major cloud suppliers.

large quantity of data, a uniform procedure of data collection, aggregation, transmission, storage, processing and visualization must be defined. As shown in Figure 1.2, major public cloud suppliers, such as Amazon, Microsoft and Google, have provided infrastructures to satisfy the increasing requirement for IoT support. Moreover, the effort to support IoT has been extended from the cloud platform to the edge device level.

However, there are still a number of obstacles and open challenges in front of the industry and hinder us from reaching a data-centric IoT framework that can be adapted to a broad category of application domains and scenarios.

- First, IoT originates from a multitude of established applicationspecific standards and protocols. As a consequence, the interoperability and compatibility between different link layer protocols, subsystems and back-end services pose a significant challenge to the establishment of a universal IoT framework. This communication challenge has not been tackled by the cloud suppliers as their efforts are mainly put into software as a service (SaaS) and platform as a service (PaaS) to enhance the computing and intelligence abilities. Cloud suppliers can hardly reach the communications among end devices due to the heterogeneity of hardware and protocols.

- Second, the application scenarios and applied technologies in IoT are diverse, therefore, the device commissioning, communication, authorization, as well as identity registration and management can hardly be integrated into a uniform security scheme to mitigate the rapidly growing device compromises and other security issues.

- Last, with novel machine learning and data mining techniques springing up, one of the ultimate goal of IoT is to harvest intelligence from 
the large volume of data in the IoT world. However, it is to a large extent restricted by the heterogeneous data format, speed, and storage mechanism from different applications and fields.

Therefore, in the context of IoT, a more feasible approach is to find out a general framework that caters to a category of application scenarios that share the same performance requirements while still maintaining the generality, as pointed out by [5].

\section{Start-of-the-art}

Many efforts have been put into pursuing a general IoT solution that can be adapted to multiple use cases or application areas.

To ease the interoperability challenges, the industries have had a consesus to utilize Internet Protocol (IP) to bridge the gaps, and the migration towards native Internet Protocol (NIP) connectivity has been observed in the majority of the traditional standards, such as BACnet/IP, KNXnet/IP, HART IP, Modbus TCP/IP, ZigBee IP, 6LoWPAN-over-BLE [7]. However, the standardization efforts are still fragmented in isolated areas.

In parallel, cellular networks have been regarded as a potential alternative to address the requirements of the four IoT segments proposed by the telecommunication industry[3]. Nevertheless, it still falls short due to three aspects. First, the broadband support in the cellular network highly relies on the 5G standard, which demands large scale of newly deployed network infrastructures which can hardly become a widespread solution in a short term. Second, the subscription cost can be a serious burden to users considering the large quantity of the deployed low power and low data rate sensors and actuators. Last, the compatibility with the massively deployed devices that only have traditional communication protocol interfaces is another challenge to conquer, as upgrading cannot be completed in a short period due to the cost and time limitation.

Some recent researches on application-oriented and service-oriented IoT frameworks are analyzed in Paper I. They achieved their specific goals but none of the frameworks can be generalized as a universal IoT framework for massive IoT and broadband IoT usages due to the lack of flexibility, reliability, availability, and security.

\subsection{Aim and Contributions}

This thesis aims to propose a data-centric IoT framework based on public cloud platforms (e.g., the Azure cloud), to face the widespread use cases in massive IoT and broadband IoT segments. It features with three promising communication protocols, namely WiFi, Thread and LoRaWAN, to satisfy both wired and wireless, wide band and narrow band, low data rate and high 
speed as well as single-directional and bidirectional communication requirements. Profiting from the evolutionary maturity of public cloud platforms, this framework is able to tackle the aforementioned interoperability, device management and data consistency challenges encountered by the industry in IoT practice. The contributions of this thesis are as follows.

1. Proposed a data-centric IoT framework exemplified with three most representative protocols to cater to the local area network (LAN), personal area network (PAN) and wide area network (WAN) use cases.

2. Eased the interoperability, co-existence, device management and data consistency challenges in the IoT practice by taking advantage of the Azure cloud infrastructure.

3. Substantiated the framework by a case study of applying the framework into the green plant wall industry so as to provide a reference implementation and prove the feasibility.

4. Proposed three different visualization methods for stream and historical data and applied neural network-based anomaly detection methods to environmental data to realize predictive maintenance of indoor climate, which showcases the data mining and intelligence abilities of the framework.

\subsection{Thesis Outline}

The reminder of this thesis is organized as follows. Chapter 2 describes the data-centric framework based on the Azure cloud platform. Chapter 3 introduces a security assessment taxonomy for wireless communications and analyzes the security aspects of the communication protocols and the cloud platform adopted in the framework. Chapter 4 exemplifies the framework with a cloud and IoT-powered green plant wall and exhibits the applicability of the proposed framework into industrial production. Chapter 5 summarises the included publications and Chapter 6 concludes the thesis. 



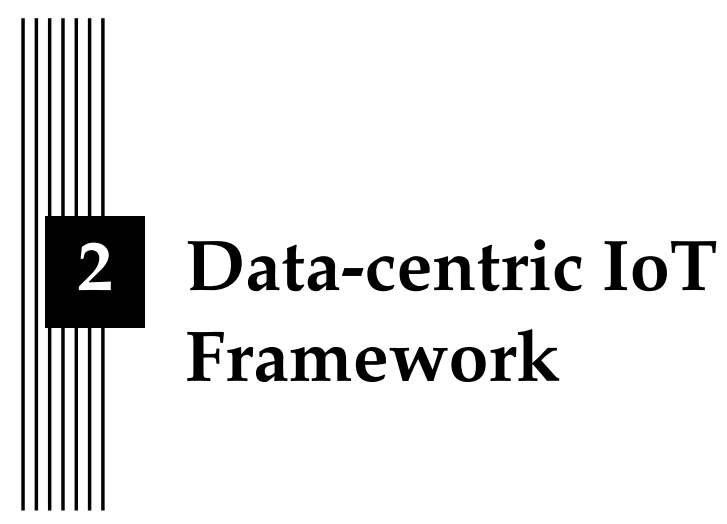

In this chapter, the details of the proposed data-centric IoT framework are introduced. The infrastructures in the cloud platform and the local field networks are described, respectively. A uniformed device model and data model are highlighted to facilitate the deployment of the framework.

\subsection{Background}

\section{Public Cloud Platform}

Benefiting from the rapid advancement of cloud technologies, cloudbased applications and services have dominated the market whereas the gradually maturing commercialization of public cloud platforms is enabling society to share the success of cloud computing. A trend has been observed that commercial companies, public organizations, universities and private persons are prone to migrate their applications, services and data storages to public cloud platforms, such as the Azure cloud, Amazon web service, or Google cloud platform [8]. Due to the rapid annual growth of connected IoT devices, providing fundamental support and seamless integration for IoT use cases are inevitable for cloud suppliers. Employing public cloud platforms instead of developing a private cloud in the IoT scenario has substantial benefits. With the aid of a public cloud platform, IoT practioners are awarded the flexibility to combine demanded services, e.g., device management, data storage, processing, analytics and visualization, etc., while avoid- 
ing the necessity to build a private platform. Profiting from the massive deployment of hardware in data centres, the total cost of utilizing a public cloud is lower than the cost of implementing their own infrastructure from scratch. The professional knowledge and experiences of public cloud suppliers are significant to guarantee the quality of services (QoS). The widespread distribution of data centers throughout the world ensures scalability of services and businesses to a large extent. Necessary redundancy of public cloud platforms guarantees high reliability since disaster recovery is usually a fundamental promise. Furthermore, in IoT use cases, security is always a prioritized concern because sensitive data leakage may incur severe consequences that can strongly affect user confidence on services. The security aspect is another merit highlighted by the public cloud platform throughout the entire stack, including software, infrastructure, storage and network[9].

Taking these merits in to account, we consider to adopt public cloud platforms to constitute the cloud end to consolidate the data-centric IoT framework. More specifically, the Azure cloud platform is employed to exemplify the proposed framework.

\section{WiFi Protocol}

The WiFi protocol is based on IEEE 802.11 standard. WiFi has become the most popular wireless local area network standard since is was released in 1997. After several years' iteration in development, the performance of WiFi such as capacity, throughput, coverage and power consumption have been greatly elevated and appreciated by the market, which makes WiFi the undoubtedly predominant wireless Internet access technology. The prevalence of WiFi is the cornerstone to cultivate innovative applications in various fields such as smart home, wearables, virtual reality (VR), augmented reality (AR). The next generation $\mathrm{WiFi}$, namely $\mathrm{WiFi} 6$, which characterizes higher data rate, larger network capacity, and better power efficiency has become an anticipated step in the IoT evolution[10].

\section{Thread Protocol}

Thread is a newly emerging mesh networking protocol that is designed for building automation networks (BAN). It adopts the IEEE 802.15.4 standard as the physical and media access control (MAC) layers while providing native support to IPv6 connectivity by introducing a 6LoWPAN layer between the MAC layer and the network layer[11]. With native-IP support, Thread promotes the building automation industry to benefit from massive innovations evolving in the Internet world. Guaranteed low latency [12] and low power consumption [13] are additional merits promised by the Thread standard. As a personal area network, a single Thread network is capable of supporting 32 routers and more than 16000 end devices, which is suffi- 
ciently large for ordinary sensing and actuating purpose. Compared to other mesh network protocols, e.g., ZigBee and Bluetooth Mesh, Thread outperforms the rest in terms of throughput, latency and reliability in either small or large networks[14].

\section{LoRaWAN Protocol}

LoRaWAN[15] is an rapidly progressing low power wide area network (LPWAN) protocol specifically designed for low power or battery-driven devices that are expected to be operating for more than a decade. LoRaWAN features long range communication, end-to-end security, mobility and positioning capabilities, low data rate and ultra-low power consumption. It has been used in various areas such as agriculture, environment monitoring, healthcare and logistics, etc. LoRaWAN theoretically supports bidirectional communications. However, the majority of the use cases only adopt the uplink communication while down-link communication is rarely used, due to the lack of a centralized consensus mechanism in the design of LoRaWAN protocol[16].

\subsection{Framework Overview}

The proposed data-centric IoT framework for massive IoT and broadband IoT is depicted in Figure 2.1. The framework is consisted of the local field and the cloud platform two parts. In the local field, connectivity among sensors, actuators and gateways are established using WiFi, Thread and LoRa communication protocols so as to cover typical IoT applications that demand local area, personal area and wide area networks support. LAN extends the Internet to a local field, which enables service to both low data rate and broadband applications, e.g., smart electronics and surveillance system. PAN is designed for low data rate and low power devices that are used within a person's living space, such as building automation systems, wearables and e-health systems. WAN supplies network infrastructures in a wider scope, mainly adopted in farming, logistics, metering or in the smart city paradigm. Large volume of data are continuously collected from sensory devices installed in the $\mathrm{WiFi}$, Thread and other wire-based networks, and then forwarded to the cloud via a WiFi access point to ensure high data rate transmission and bidirectional communication. Alternatively, an edge device can be deployed in the local field to enhance local processing abilities such as data cleaning and local computing. Messages of low power and low complexity sensors that are deployed in a wide area are transmitted using the LoRa network, taking advantage of the distinguishing low power consumption and long range communication features.

The cloud part is comprised of data processing, storage, and presentation and management components. The core unit in the cloud platform is an 


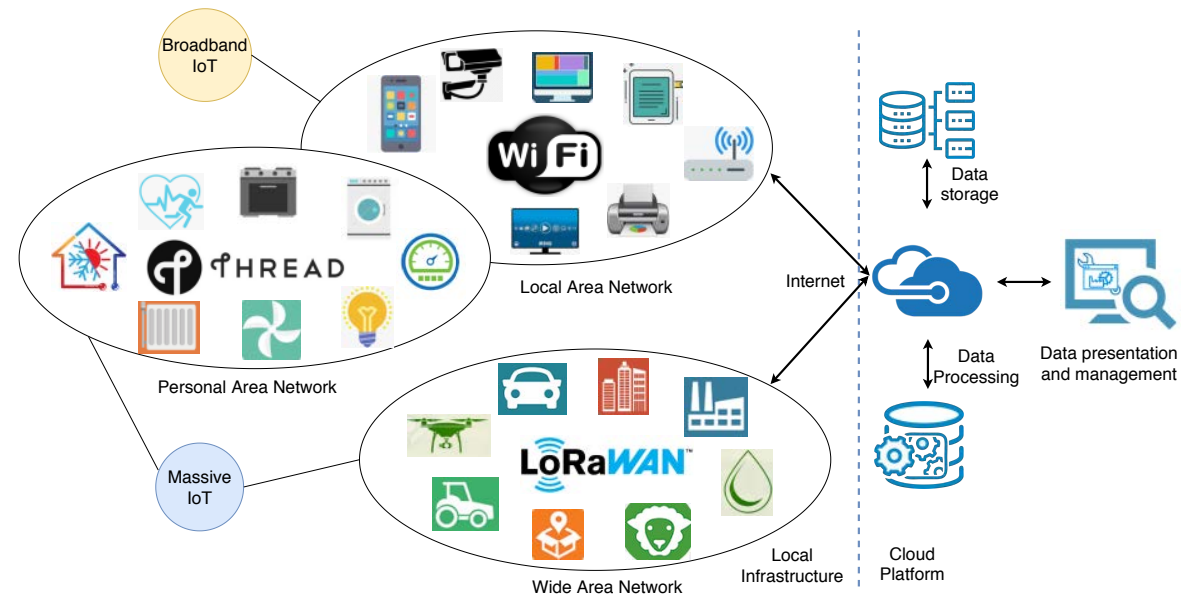

Figure 2.1: Overview of the data-centric IoT framework for massive and broadband IoT.

IoT Hub service which is responsible for device provisioning, device identity management and data routing. A series of other services that are implemented with applications can be flexibly integrated into the IoT Hub infrastructure as plugins, depending on practical needs. A cloud gateway in an IoT Hub is in charge of authentication and data routing for all incoming messages that are transmitted from different devices and locations, before further processing, storage and visualization services are performed. A web application is developed and hosted by a container service to realize real-time visualization, historic data display and administrative management functions for end users.

\subsection{Azure Cloud Infrastructure}

The proposed framework capitalizes on the IoT Hub infrastructure in Azure to realize a centralized IoT management service, to address the challenges in respect of interoperability, device management and data format consistency. The service connectivity are shown in Figure 2.2.

\section{IoT Hub}

IoT Hub sits in the center of the cloud platform in the framework, which offers fully-managed services such as device provisioning, authentication, identity management as well as data ingestion and routing. IoT Hub supports secure and bidirectional communications with millions of IoT devices using HTTPS, advanced message queuing protocol (AMQP) or message queuing telemetry transport (MQTT) protocols. It maintains direct connec- 


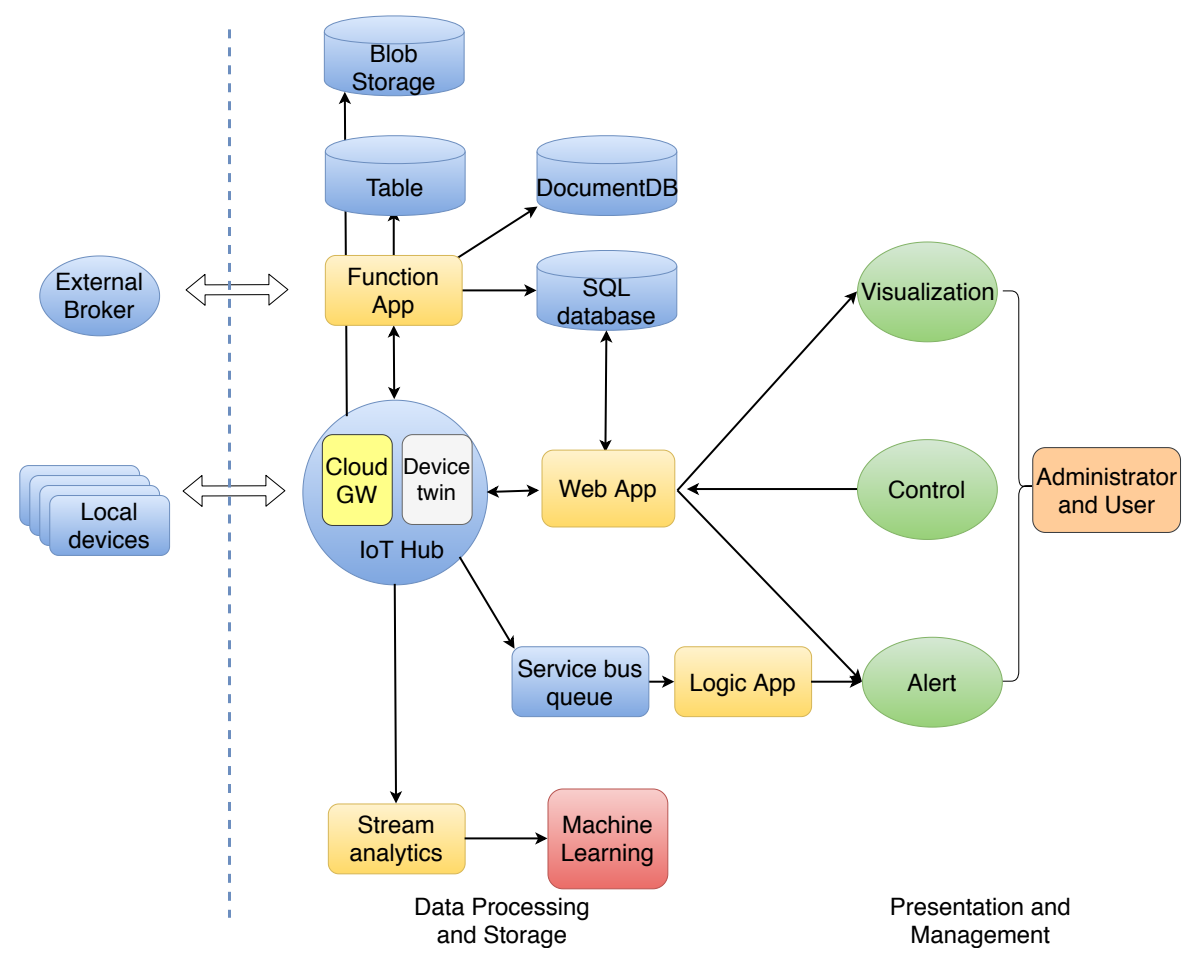

Figure 2.2: Cloud service connectivity of the data-centric IoT framework.

tions to IP-capable devices and also reserves the possibility to establish connections to low-power and resource-constrained devices, or devices with other protocols via a field gateway[17].

\section{Peripheral services}

In the framework, a series of peripheral services are seamlessly plugged into the IoT Hub infrastructure to accelerate data analytics, storage or presentation of collected telemetries.

Function service provides the flexibility to execute a specific task with a small piece of code, without developing a whole application or project. Functions can be written in a number of high level programming languages, such as C\#, Node.js and Python, etc. In the framework, function services behave as adaptation units between data ingestion and storage services by taking away the constraints of heterogeneous data sources and destinations. With function services, data from different devices or locations can be stored to different databases according to their contents or properties.

Data storage service is largely depending on the type of received data. This framework supports four types of storage services in IoT use cases. SQL 


\begin{tabular}{|c|c|c|}
\hline PROTOCOL & LAYER & FUNCTION \\
\hline WiFi & $\begin{array}{l}\text { Link layer } \\
\text { bidirectional }\end{array}$ & $\begin{array}{l}\text { Create local area network; Connect an } \\
\text { edge device to the cloud; Connect WiFi } \\
\text { devices with an Edge device }\end{array}$ \\
\hline Thread & $\begin{array}{l}\text { Link layer } \\
\text { bidirectional }\end{array}$ & $\begin{array}{l}\text { Create personal area network; Connect } \\
\text { Thread end devices to a Thread Border } \\
\text { Router }\end{array}$ \\
\hline LoRaWAN & $\begin{array}{l}\text { Link layer } \\
\text { single- } \\
\text { directional }\end{array}$ & $\begin{array}{l}\text { Create low power wide area network; } \\
\text { Connect LoRa devices to a LoRaWAN } \\
\text { gateway }\end{array}$ \\
\hline Ethernet & Link layer & $\begin{array}{l}\text { Connect a WiFi access point to the } \\
\text { cloud server; connect LoRa backend } \\
\text { server to the cloud }\end{array}$ \\
\hline MQTT & $\begin{array}{l}\text { Application } \\
\text { layer }\end{array}$ & $\begin{array}{l}\text { Enable data exchange between an edge } \\
\text { device and an MQTT-SN (MQTT for } \\
\text { sensor networks) gateway for Thread } \\
\text { end devices, between an edge device } \\
\text { and WiFi devices, and between edge } \\
\text { devices and loT Hub }\end{array}$ \\
\hline $\begin{array}{l}\text { MQTT- } \\
\text { SN }\end{array}$ & $\begin{array}{l}\text { Application } \\
\text { layer }\end{array}$ & $\begin{array}{l}\text { Enable data exchange between an } \\
\text { MQTT-SN gateway and Thread end } \\
\text { devices }\end{array}$ \\
\hline HTTPS & $\begin{array}{l}\text { Application } \\
\text { layer }\end{array}$ & $\begin{array}{l}\text { Enable data exchange between an edge } \\
\text { device and IoT Hub; enable file up- } \\
\text { load to blob storage service (broad- } \\
\text { band support) }\end{array}$ \\
\hline AMQP & $\begin{array}{l}\text { Application } \\
\text { layer }\end{array}$ & $\begin{array}{l}\text { Enable data exchange between an edge } \\
\text { device and loT Hub }\end{array}$ \\
\hline CoAP & $\begin{array}{l}\text { Application } \\
\text { layer }\end{array}$ & $\begin{array}{l}\text { Enable data exchange among Thread } \\
\text { devices }\end{array}$ \\
\hline UART & N/A & $\begin{array}{l}\text { Enable communication between a } \\
\text { Thread network co-processor and a } \\
\text { host device to constitute the Border } \\
\text { Router }\end{array}$ \\
\hline $\begin{array}{l}\text { Other } \\
\text { wired } \\
\text { protocols }\end{array}$ & N/A & $\begin{array}{l}\text { Enable communication between field } \\
\text { sensors/actuators and an edge device }\end{array}$ \\
\hline
\end{tabular}

Figure 2.3: Communication standards used in the framework.

database is used for storing relational data while DocumentDB features the light weight, fast query speed and simple data format, e.g., JSON format. Table storage is reserved for storing table structured data. Blob storage is deployed for storing unstructured data such as uploaded video, image, raw data file, or device specific files.

Visualization service is served by Azure as an out-of-box tool for users, e.g., the PowerBI service. This framework relies on a web application-based human-machine interface (HMI) to leave more freedom for customized visualization methods according to distinguished data sources.

Intelligence services in this framework include processing collected data, inserting analytic and logic units, and applying machine learning al- 


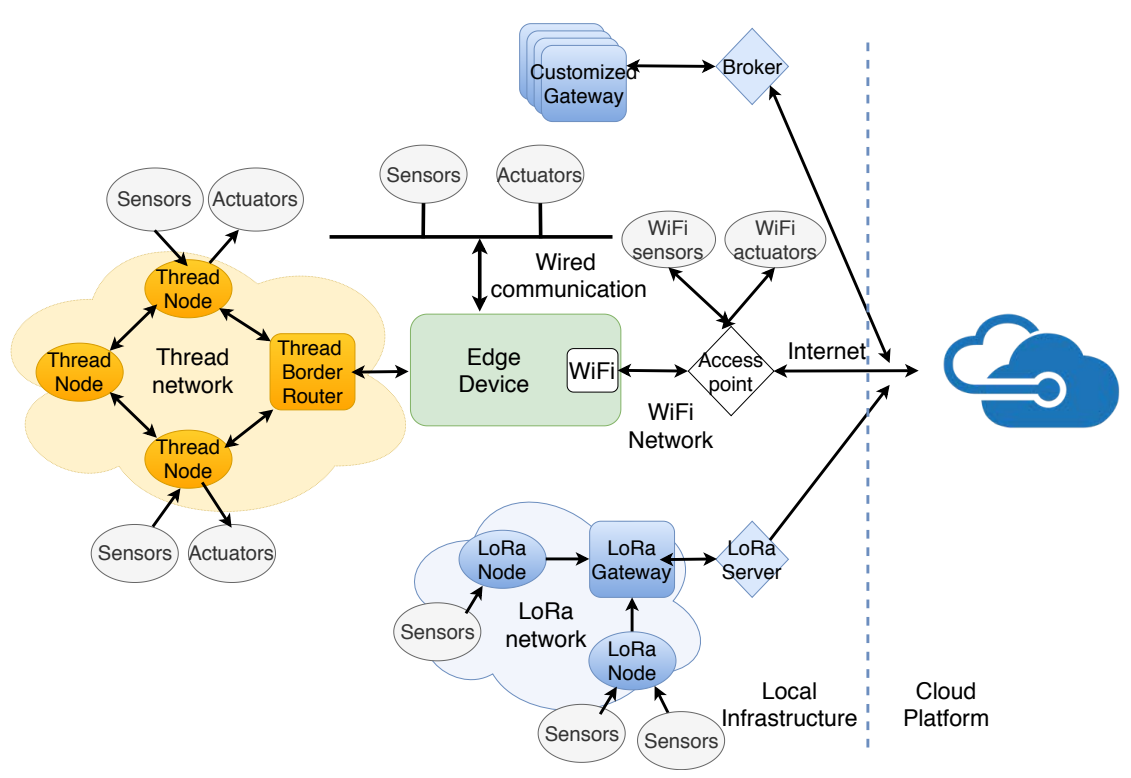

Figure 2.4: Local networks connectivity.

gorithms. For instance, stream analytic services combined with built-in machine learning services can be integrated to accelerate the time series data analytics. A logic application is able to enable alert functions. Besides, more advanced and flexible user-developed machine learning and deep learning models can be deployed together with the web application to realize customized intelligent functions.

\subsection{Local Infrastructure}

\section{Communication standards}

In the local field, several data link layer and application layer communication protocols are employed to enable interoperability among devices from different networks. They are listed in Figure 2.3 with detailed function descriptions.

\section{WiFi Network Connectivity}

As shown in Figure 2.4, WiFi network is the backbone network in the framework to provide connectivity between the local infrastructures and the cloud. Due to its native-IP support, devices with a WiFi interface can directly communicate with the cloud gateway using any of the supported messaging protocols via an access point. In parallel, the message exchange between resource-limited devices and IoT Hub can be bridged by the edge device. 


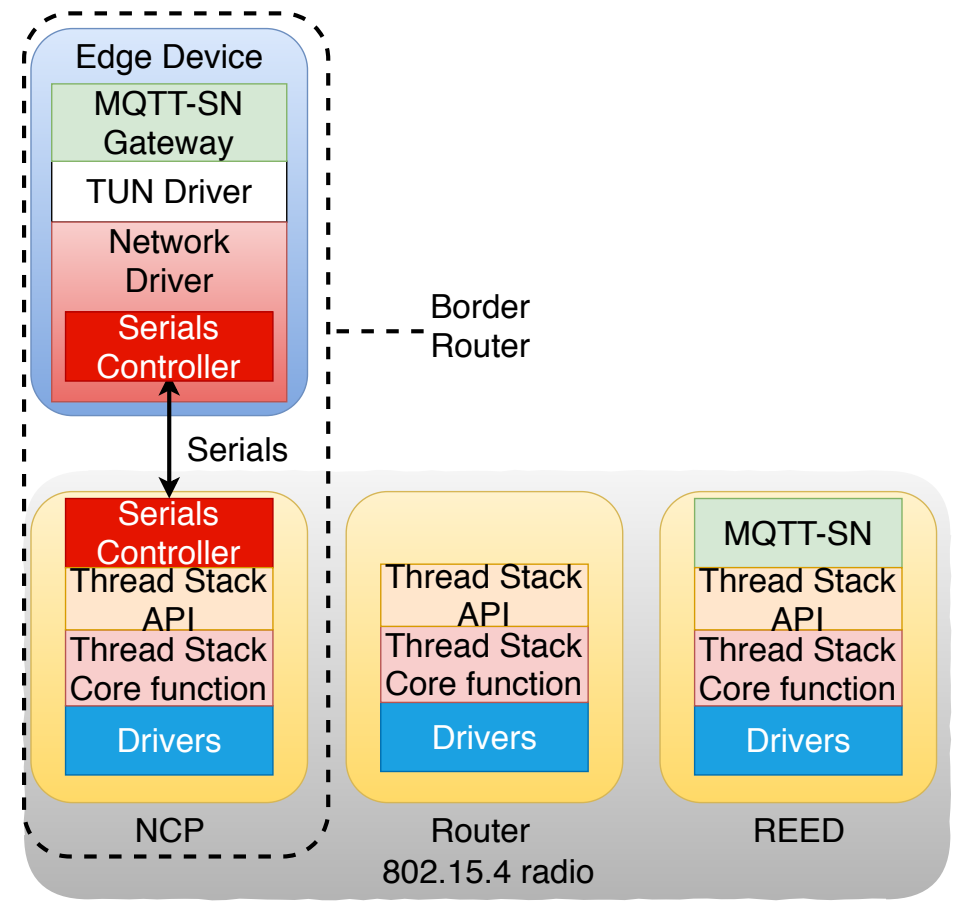

Figure 2.5: Thread and edge device connectivity.

The bidirectional communication traffic between the edge device and WiFienabled sensors is carried by light-weight application layer protocols such as constrained application protocol (CoAP) and MQTT to satisfy the low-power requirement.

\section{Thread Network Connectivity}

Thread is introduced into the framework as a complement to the WiFi network. Benefiting from the native support of building automation by Thread, this framework is able to seamlessly incorporate building automation systems using the same infrastructures in the cloud.

The connectivity between the Thread network and the edge device is enabled by a Thread border router, which is in charge of adapting IP traffics to Thread packets and vice versa. The details of the connectivity are shown in Figure 2.5. A host-NCP (network co-processor) architecture is used to realize the border router functions. A Thread network co-processor implements the full Thread stack, i.e., Thread drivers, Thread stack core functions, and Thread stack APIs, while the application layer is hosted on the edge device. As a agent of the edge device, the network co-processor is able to create a Thread mesh network and listen to the network traffics through the Thread 
interface. After network creation, other Thread devices, such as routers and router eligible end devices (REED) can be commissioned to join the network using the standard Thread commissioning procedure. The communication between the edge device and the network co-processor is via a serial bus that is managed by serial controllers in both devices. All the packets from the Thread network are routed to application layer programs in the edge device through a network driver and a tunnel driver. The network driver is a user-space program that provides native IPv6 interface to the network coprocessor device while tunnel driver is in charge of packet routing between different user-space programs.

In the application layer, the interaction between a Thread device and the edge device is through the MQTT protocol and an MQTT broker, similar to WiFi nodes. Specifically, a lightweight MQTT protocol, namely MQTT for sensor networks (MQTT-SN) is utilized to enable Thread devices to communicate in the same way as using MQTT, which largely improves the interoperability among the local field devices.

\section{LoRaWAN Connectivity}

As seen in Figure 2.4, a LoRaWAN network is involved in the framework to provide services in a wider scope than LAN and PAN to perform metering, tracking or other monitoring tasks. The present framework only utilizes the single directional up-link communication of LoRaWAN, while the down-link is neglected for reliability consideration. (According to industrial reality, reliability down-link service needs very dense deployment of gateways.) The LoRaWAN services are recommended to be built upon commercial LoRaWAN operators' network due to the large scale deployments of gateways and superior compatibility between LoRaWAN servers and public cloud platforms.

\section{Edge Device}

Figure 2.6, depicts the structure of an edge device that behaves as a local hub where all device telemetries are aggregated and then forwarded to the cloud. Meanwhile, cloud to device messages are first transmitted to the edge device and then relayed to target devices. In this framework, an edge device is compromised of an standalone application, an edge broker, an adaptation server, an Internet module and an edge computing unit, which consolidate the fundamental functionalities.

The Standalone application is running in an isolated process in parallel with other components to interact with sensors and manipulate actuators that are directly connected to the edge device. A standalone application has its own identity registration in the cloud to represent the edge device itself. 
A broker is a message server that delivers messages from publishers to subscribers, which is vital to enable the interoperability between the edge device and Thread/WiFi devices. For all Thread and WiFi nodes that are not able to establish a direct link to the cloud, a series of device-specific topics shall be registered in the broker server for each individual device, which include telemetry, command and property topics. In this approach, end devices are able to transmit sensory telemetries and receive remote commands by publishing content to the telemetry topics and subscribing to the command topics. Property topics are used to modify end device local settings or configurations which shall be always synchronized to the device properties in the cloud.

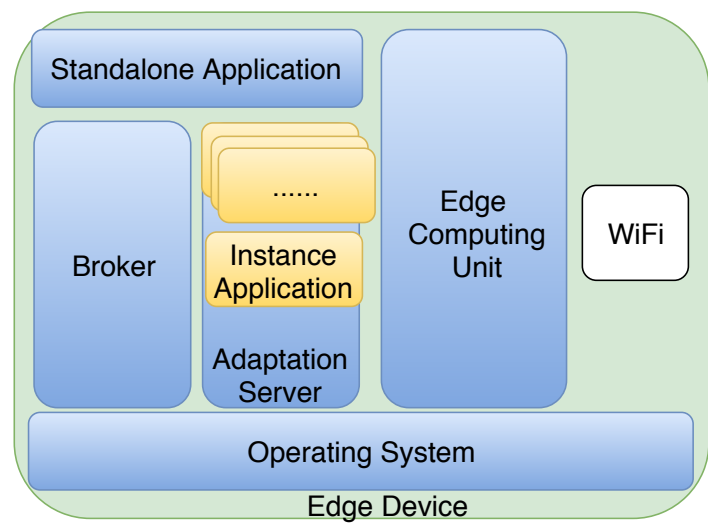

Figure 2.6: Edge device structure.

The adaptation server maintains a couple of application instances. Each of them represents an end device and has its own device identity object, which is always synchronized to the corresponding device identity in the cloud. An application instance interacts with the corresponding device through the broker server. It subscribes to relevant device telemetry topics to get updated sensory data and invokes direct methods by publishing method to command topics. The details of this procedure is described in the Data flow subsection.

The Internet module is required to enable the edge device to establish communication channel with the cloud platform. In most cases, a WiFi interface is equipped.

The Edge computing unit releases the potential of the edge device and bring intelligence closer to the local infrastructures. It can perform a local gateway for device provisioning, data cleaning or light-weight analytics, and greatly reduce the amount of data being transferred to the cloud. 


\section{External broker}

An external broker is exploited in the framework to improve the compatibility with other communication protocols, It is especially meaningful for traditional field network protocols that have been widely deployed. The sensory messages can be published to the external broker server, and then fetched by a function application that is running in the cloud and has subscribed to the broker. The data can be conveniently stored into the same database as data generated from other first class devices.

\subsection{Device Model}

The unified device model of this framework is shown in Figure 2.7, which is applied to all the registered devices. Upon device provisioning, all the device-specific information, e.g., device ID and authentication key, are stored in the cloud device identity registry, regardless of the hardware difference. The device management procedures involve three patterns: property update, bidirectional message and direct method. Each device corresponds to two identical conceptual objects, namely device twin, in the registered IoT Hub and in the local device. A device twin contains device tags, desired properties and reported properties. By synchronizing local desired properties to the cloud desired properties and updating the latest local reported properties to the cloud reported properties, the device twins are always identical. Device to cloud (D2C) messages and cloud to device (C2D) messages are natively supported by IoT Hub to enable telemetry and notification delivery. Direct method is an approach to instantly invoke local commands in the end device to execute. With these device twin objects and three device management patterns, this framework can treat all devices with a single device model.

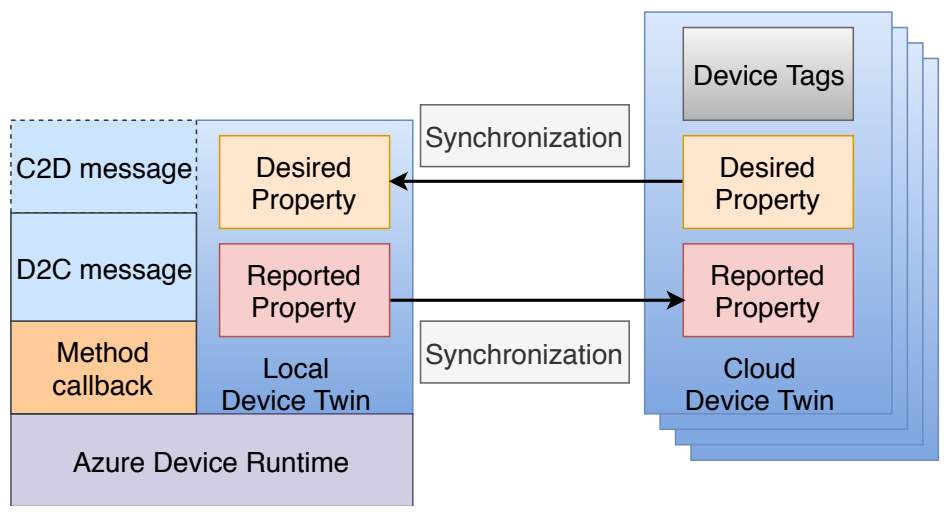

Figure 2.7: Data flow of Thread and WiFi devices in the framework. 


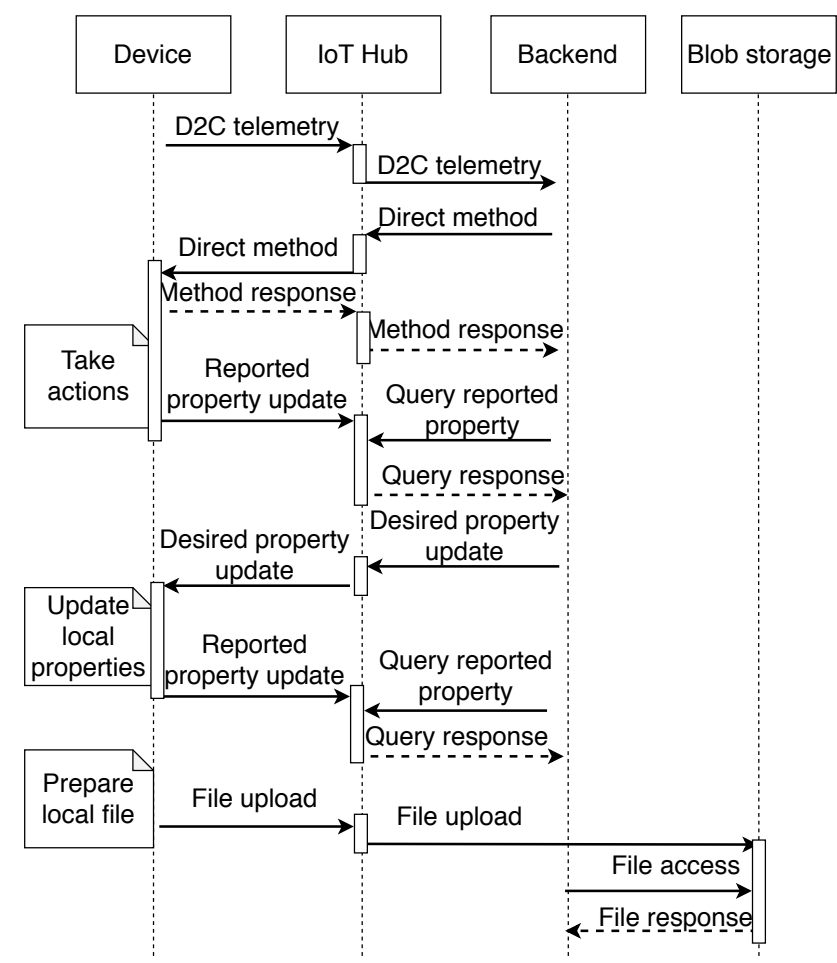

Figure 2.8: Data flow of standalone application in the framework.

\subsection{Data Model}

\section{Data Format}

To ensure data format consistency, the JavaScript Object Notation (JSON) encoding format is adopted in this framework to encode messages. JSON characterizes with its ubiquitousness and broad library or platform-inherent support[17]. Incoming JSON formated messages can be automatically routed to different services according to message contents by IoT Hub. Therefore, in this framework, all the sensory messages and device twin objects are encoded in JSON to guarantee interoperability.

\section{Data Flow}

In this framework, the data flow between the cloud and end devices involves D2C telemetry, C2D direct method, desired property update, reported property update, and unstructured data upload.

The data flow of a standalone application is shown in Figure 2.8. A D2C telemetry is a JSON encoded message mainly used for reporting local sen- 


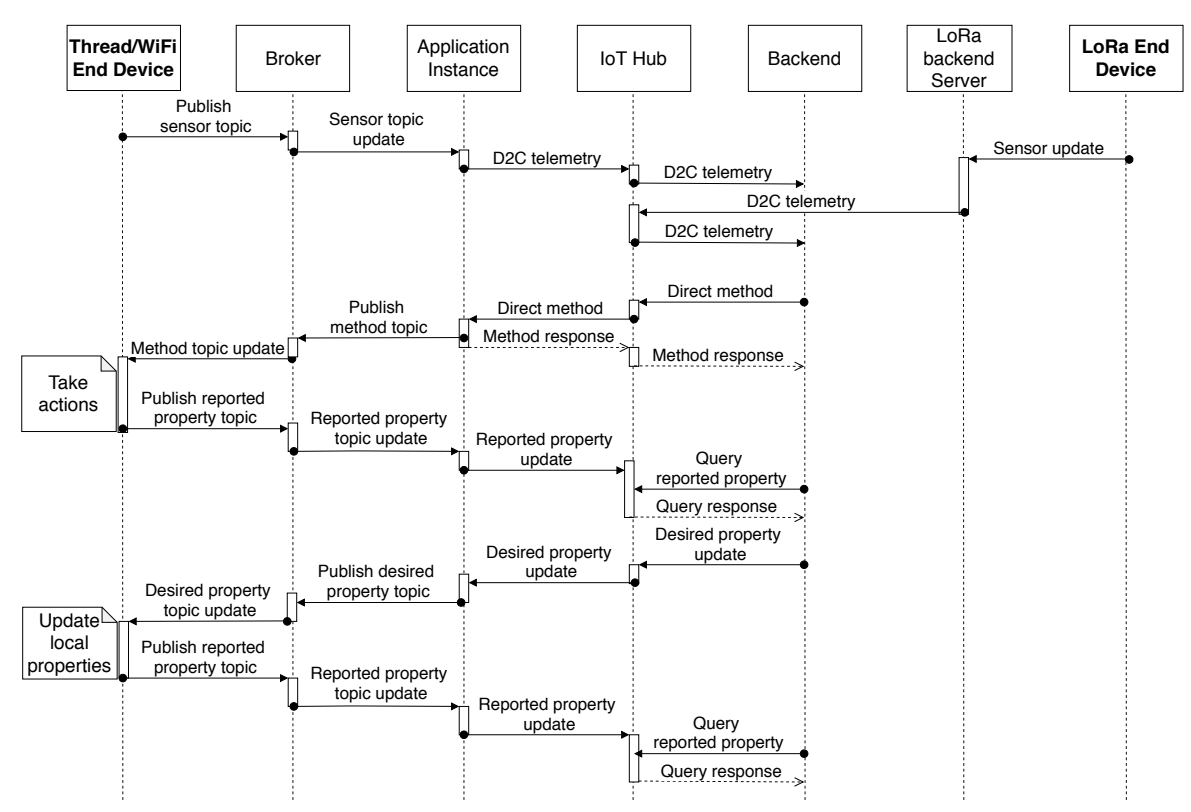

Figure 2.9: Data flow of Thread and WiFi devices in the framework.

sory status. The backend can manipulate a local device either by sending a direct method to the device to trigger an action, or by requesting a desired property update to change device local settings. In either case, the end device will execute the corresponding task and then inform the backend of the execution results with a reported property update. These four categories of data flow can satisfy the need for monitoring and remote control in the Massive IoT use cases. Unstructured data upload caters to the Broadband IoT requirement which enables a large amount of unstructured data transferred to the cloud blob storage.

Figure 2.9 depicts the data flow of Thread, WiFi and LoRa nodes.A Thread or WiFi end device publish sensor data to the MQTT broker so that the corresponding application instance is able to construct D2C telemetries for the Thread/WiFi node upon receiving a notification from the broker. Sensor data from a LoRa end device are first reported to a LoRa backend server, which encapsulates received data into D2C messages and transmits them to the cloud later on. Similarly, a direct method or desired property update is also relayed by a application instance and then delivered to the Thread/WiFi end device using MQTT broker-based notification. Particularly, file upload data flow is only enabled by standalone applications due to its direct access to broadband WiFi network while an MQTT-based communication is not optimal for file transfer. 
A reference implementation of this framework is presented in Paper I as a proof-of-concept. 


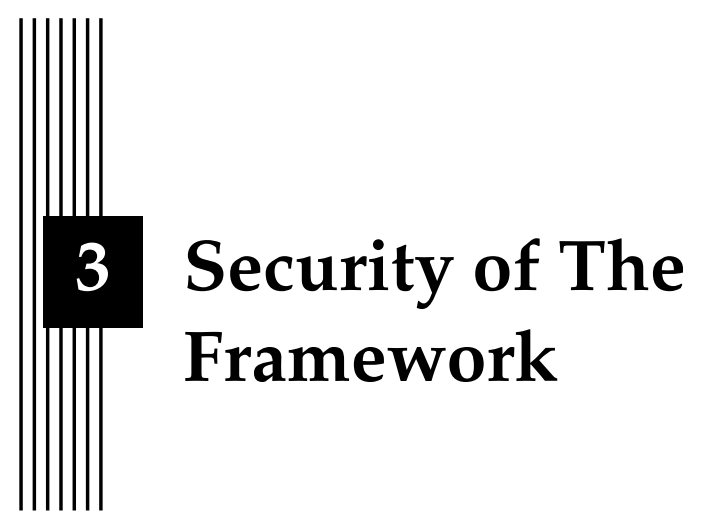

Security is always a major concern of practioners in the IoT practice. In the framework, the security performance is initially considered and highly prioritized which covers both local communications and interactions in the cloud. In this chapter, a security assessment taxonomy is presented as a tool to evaluate the security aspects in communications in building automation networks (BAN) belonging to a building automation system (BAS). It can also be extended as a security measurement for massive IoT and broadband IoT use cases. The security performances of Thread, WiFi and LoRaWAN are discussed, then followed by a security analysis of the cloud platform utilized in the framework.

\subsection{Security Relevant Characteristics}

- Limited resource availability [18][19][20]: Sensors and actuators in BANs are usually energy constrained embedded devices with limited computational resources, therefore, computationally inexpensive cryptographic algorithms and protocols have to be used [19].

- Diverse topologies [18][20] A single BAN can consist of as many as thousands of devices using unicast and multicast communications to provide services. Due to the diverse topologies, the network structure and the security solutions need to be scalable and adaptive [20]. 


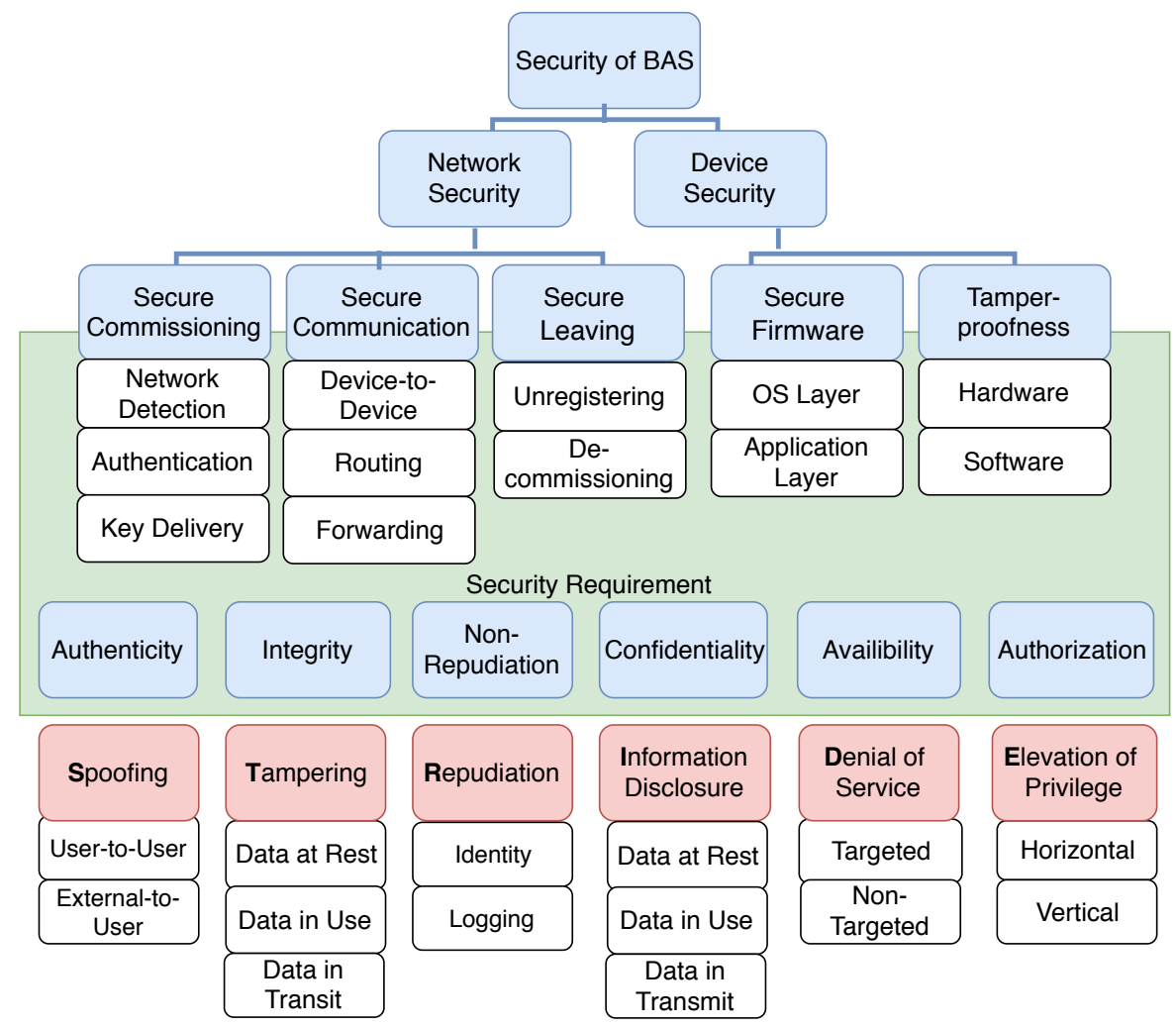

Figure 3.1: Proposed Security Analysis Taxonomy for BASs.

- Physical access [19] Devices in a BAS typically operate in an open environment, hence they are exposed to the risk of physical access that can cause sensitive data or network credentials leakage [21].

- Continuous maintenance [19] BANs can have a lifespan as long as a decade. Hence, firmware upgrades are essential for deploying new functionalities and for protecting the devices and the system from new attacks. Firmware upgrades need, however, to be performed in a secure manner so as to avoid system compromise.

\subsection{Taxonomy for Security Assessment}

\section{Five Phases of Interaction}

Secure Commissioning Commissioning in a BAN is the process through which a device locates the correct BAN that it should attach to, mutually authenticates with the authorized commissioner in the BAN, it is entrusted 
with network data and credentials, and finally it establishes a secure communication channel with other entities in the network. In order for commissioning to be secure, a BAN standard has to guarantee security during network detection, authentication and during the delivery of network secrets. It has to protect the network from spoofing, man-in-middle and denial-of-service attacks and it should not leak any secret about the network to an unauthorized third party.

Secure Communication After commissioning, a device implements or contributes to functions through communicating over the established channels using the network credentials. In order for the communication to be secure, routing information exchange, message forwarding and device-to-device data delivery should be secure.

Secure Leaving Once a successfully commissioned device is forced to leave or decides to join another BAN, the removal of the device from the network (unregistering) and destruction of sensitive information about the BAN (decommissioning) have to be performed in a secure manner, as exposure of the network credentials could be used for violating the security of other BAN devices.

Secure Firmware A typical BAN device executes code stored in nonvolatile memory, i.e., firmware. The code can be divided in two levels: a low level operating system (OS) that manages hardware and provides interaction between resources and applications, and high level applications that contain functional tasks. A compromise at any of these two levels implies compromise of the device [19]. Furthermore, upgrading functional tasks of the device requires upgrading the firmware. Thus, secure OS and application code development, as well as secure configuration and firmware upgrades are essential.

Tamper-proofness A BAN device needs to be protected from compromise through tampering, as a compromised device may be used for obtaining the network secrets or for manipulating data. Protection is unlikely to be achieved by physical isolation, thus tamper protection needs to be applied on the chips and the memory inside the devices, to protect the hardware and the software.

\section{Security Requirements}

Our taxonomy formulates security requirements for all five phases, in face of threats structured according to a refined STRIDE threat model, and considers both active and passive adversaries. In the refined STRIDE model, 
we distinguish between spoofing by an internal and an external adversary, we consider tampering and information disclosure attacks against data at rest, in use and in transit, we consider repudiation attacks through affecting identity management and logging, we distinguish between targeted and non-targeted DoS attacks, and between horizontal and vertical elevation of privilege attacks.

For all five phases, security requirements in terms of authenticity, Integrity, Non-repudiation, Confidentiality, Availability and Authorization are evaluated so as to have a comprehensive analysis of the security level of a BAN. For detailed descriptions of the taxonomy, please refer to Paper II.

\subsection{Security in Thread}

Thread is known for having superior security consideration. Commissioning and security are highly prioritized in the specification of Thread. The security mechanism covers the whole life cycle of a Thread device, i.e., from a device discovering and joining the network to leaving the network. Device commissioning in Thread is protected with a datagram transport layer security (DTLS) session which fulfills online and offline dictionary attack resistance, forward secrecy and known session security. Communication in Thread is protected by an AES-CCM security suite that ensures confidentiality, integrity and authenticity. A series of novel network attacks towards Thread are also identified in [22] and it turns out Thread is robust to the majority of the identified attacks except for some radio jamming attacks that can generally affect all networks. Several security enhancement countermeasures were also proposed to reinforce the security of Thread in [22]. For more details about the security analysis of Thread network, please refer to Paper II.

\subsection{Security in WiFi}

For WiFi network, the vast majority of the devices are secured with WiFi Protected Access 2 (WPA2) encryption method, which has been found vulnerabilities, e.g., it is sensitive to key reinstallation attack (KRACK)[23]. WiFi alliance has announced the approaching of the next generation WiFi protocol, 802.11ax, with an enhanced encryption method namely WPA3[10]. WPA3 manages to mitigate the flaws in WPA2 such as offline dictionary attack and KRACK attack. Forward secrecy is another highlighted security feature of WPA3 that prevents old data being disclosed by later attacks. In general, the new WiFi release will bring security to an unprecedentedly high level. 


\subsection{Security in LoRaWAN}

In LoRaWAN, end-to-end security is enabled and two types of session keys are utilized to guarantee a secure channel established between an end device and a LoRa application server. A 128-bit AES security key is bound to each LoRa device to adopt AES cryptographic primitive with several operation modes, e.g., cipher-based message authentication code (CMAC) mode for integrity protection and counter (CTR) mode for encryption. LoRaWAN implements integrity protection in a hop-by-hop manner, i.e., one hop over the air is guaranteed by LoRaWAN protocol and the other hop between the network and LoRa server is protected by secure transport solutions such as HTTPS and VPNs[24].

\subsection{Cloud Security}

In the cloud platform where IoT data are transported between different cloud services and databases, the security is guaranteed by Azure security infrastructure which provides high levels of enhanced security, privacy, compliance, and threat mitigation practice[25].

IoT Hub uses a per device authentication manner to guarantee the isolation of security for separate devices. Moreover, each security key is bound to an access policy when it is generated, which greatly improves the security in authorization. For instance, a device bound to a DeviceConnect policy can only send D2C messages and receive C2D notifications and direct methods, but has no access to device registry or any other IoT Hub settings. Communication between IoT Hub and devices is secured with transport layer security (TLS) based handshake and encryption, which is the cornerstone of the security of the current Internet society.

\subsection{Security Summary}

By taking advantages of existing security schemes of the Azure public cloud and WiFi, Thread and LoRaWAN protocols, this proposed framework has taken up this key point in network security, though more details regarding device security such as secure operating system, secure application layer, and hardware tamper-proofness that are more relying on implementation shall be further considered. 



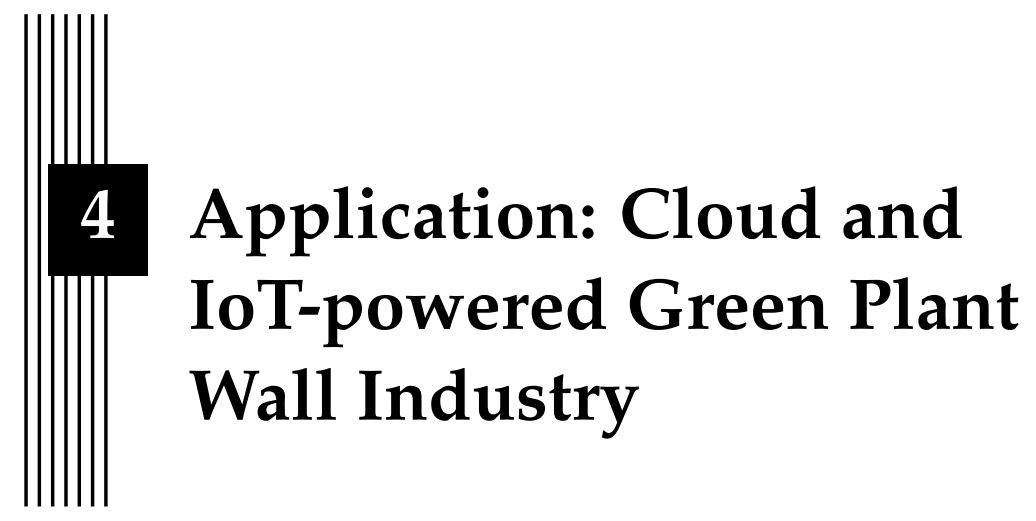

In this chapter, we adopt the proposed IoT framework to facilitate the digitalization process of the green plant wall industry with low cost and guaranteed reliability, availability, scalability and security. The implementation details are presented as a validation of the framework and a reference practice to other industries with the same needs. specifically, the solution architecture is disclosed and described in detail. Three visualization methods are proposed to better interpret the stream and historical environmental data. Furthermore, a neuron network-based anomaly detection method is benchmarked and integrated into the framework to showcase the significance of combining deep learning and big data in the IoT era.

\subsection{Green Plant Wall Industry}

A plant wall system is to grow diverse types of green plants on a vertically supported system that is attached to an internal or external wall or is designed as a standalone product. It consists of vegetation, growing medium and irrigation and drainage systems [26]. In addition to decoration, green plants contribute to indoor environments via evaporation, air purification and water retention.

There are some constraints that hinder the plant wall industry from entering the household market. First, regular management of massive plant walls is costly and time-consuming for plant wall suppliers. Second, regular service of plants is largely limited by geography. Thus, reliable and af- 
fordable remote management and monitoring solutions for plant walls are in high demand. In this type of systems, the indoor environmental parameters such as temperature, relative humidity $(\mathrm{RH}), \mathrm{CO}_{2}$ level, and volatile organic compounds (VOC) concentration, must be collected and monitored by both consumers and suppliers in real time, and suppliers with expertise in plantcare shall be able to remotely control the watering, lighting and ventilation functions.

\subsection{Solution Overview}

To tackle the aforementioned challenges, we propose and implement a remote monitoring and management solution for plant wall industry based on the data-centric IoT framework. The system overview is shown in Figure 4.1. The proposed solution consists of both a local control part and a cloud service part. In the local part, a series of environmental parameters are monitored and the data are continuously transmitted to the cloud using the WiFi protocol. Meanwhile, the control functions of watering, lighting and ventilation in a plant wall system are considered. The cloud part takes advantage of the IoT Hub infrastructure and other services, such as function applications, storages and web applications offered by the Azure platform. Via a web-based user interface, administrators and end users are able to monitor an indoor climate in real time, check historic data from a database, and update the schedules of the pump, light and fan functions, as well as invoke actuators for management purposes.

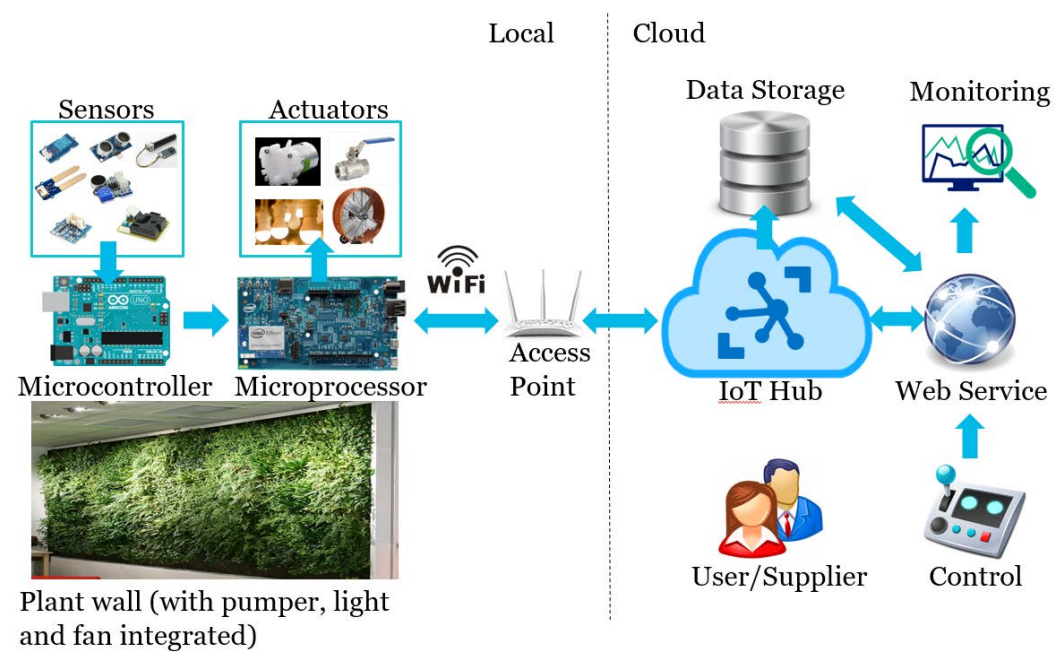

Figure 4.1: System overview of Azure cloud and IoT-based remote monitoring and management system for plant walls. 


\subsection{System Implementation}

\section{Hardware Components}

The local infrastructures of the system is shown in Figure 4.2, which details the connectivity of hardware components.

\section{Microprocessor and Microcontroller}

A microprocessor in the local part is the central processing unit that is in charge of transmitting sensor data to and receiving messages from the cloud. Moreover, it also executes control functions to take care of the plant wall. In our solution, Intel Edison is selected as the microprocessor used in the local unit. The Edison module is a system-on-chip (SoC) featuring a dual core, dual-threaded Intel Atom CPU. It has an integrated on-chip WiFi module and supports many external interfaces, e.g., UART, I2C, SPI and I2S protocols. A modern embedded Linux operating system, namely, Yocto Linux, is natively supported by Edison.

Arduino Uno is utilized in our solution as the microcontroller due to its popularity and broad support from the community. It is based on the ATmega328 chip. Arduino Uno has six analog input pins and 14 digital input/output pins, of which six pins can be employed as PWM outputs. It also supports UART, I2C and SPI communications. The rich I/Os and communication interfaces makes Arduino Uno the reliable choice for controlling the sensor readings.

\section{Sensors and Actuators}

The sensors and actuators used in our solution are as follows.

- Temperature and relative humidity sensor

- Visible and infrared light sensor

- $\mathrm{CO}_{2}$ sensor

- Particulate matter sensor

- Three channel gas sensor

- Ultrasonic sensor for water level

- 230 V AC Pump

- 24 V DC Fan

- 24 V DC LED light 


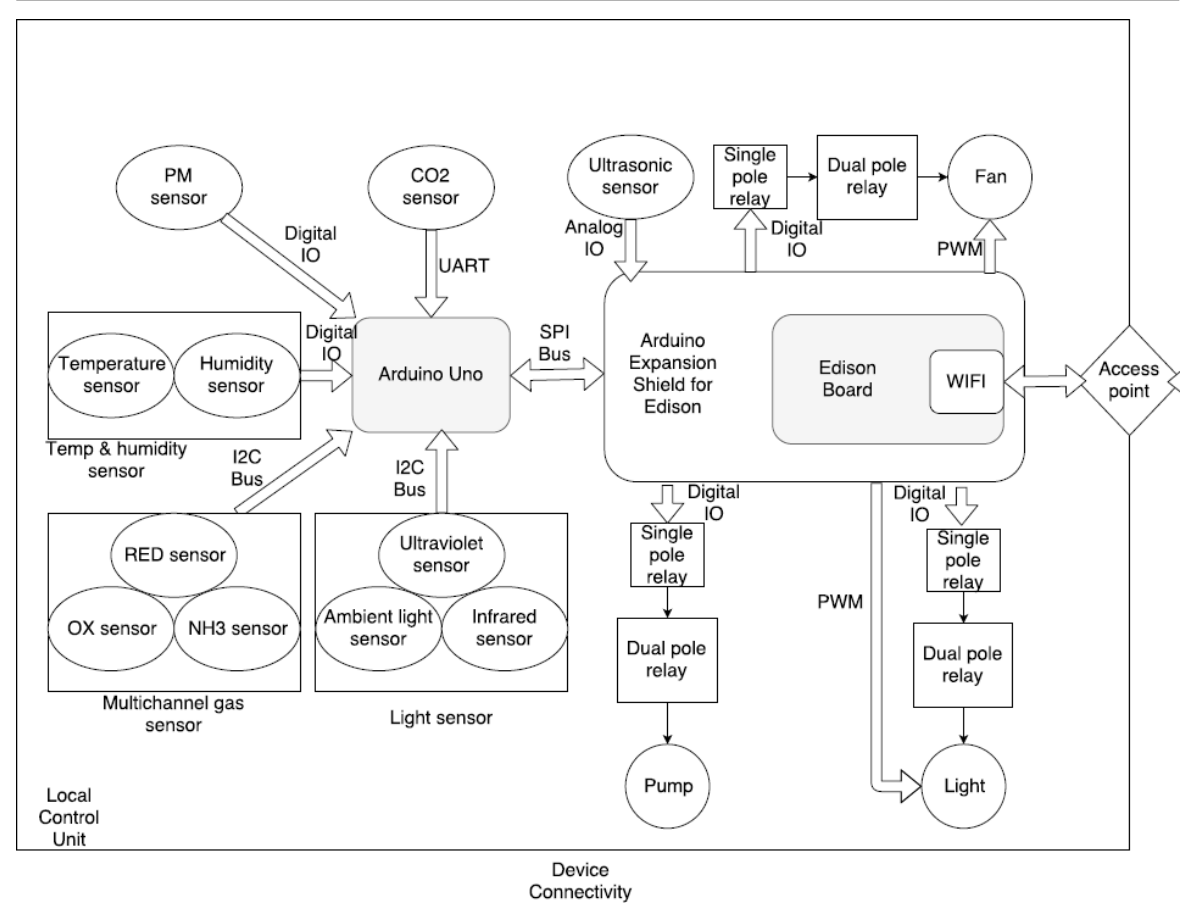

Figure 4.2: Hardware components connectivity of proposed solution for plant wall system.

\section{Software Design}

The program running on Arduino is responsible for obtaining the sensors data and transmitting them to Edison during query. The main loop is to periodically read all of the sensors every five seconds, and the results are locally stored and updated in a buffer. Once interrupted by Edison, the latest data are fed back to Edison via SPI bus.

The program running on Edison is developed based on MRAA library and Azure-iot-sdk. They are provided by Interl and Microsoft, respectively, to speed up the development procedure, ease the engineering work and improve the program soundness. The main tasks are shown in Figure 4.3. Both online and offline modes are enabled to ensure the stability of the control unit in case of loss of Internet. Figure 4.4 shows the property object used in this plant wall system. The administrators/users can manage the plant wall by modifying the desired properties in the cloud, whereas the changes are informed to the device. After receiving the notification, the device changes the local settings according to the desired properties and then update its value to the reported properties to ensure that the desired and reported properties are synchronized in the cloud. In this way can we control the fan, pump, 


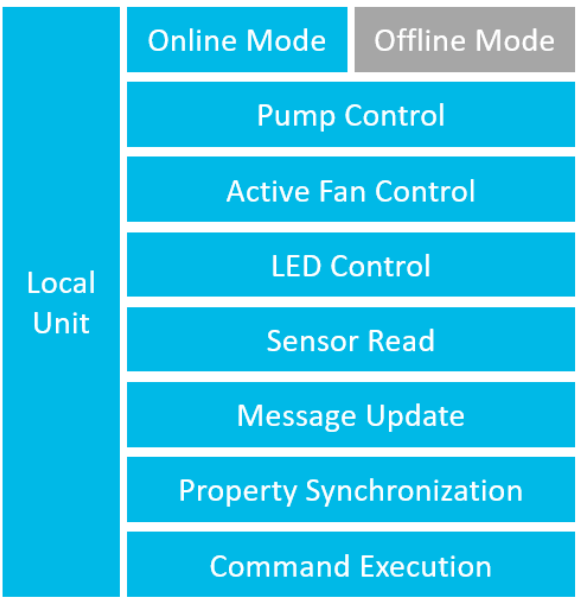

Figure 4.3: Tasks of the local unit.

\begin{tabular}{|c|c|c|c|}
\hline & & Desired Interval & \\
\hline & & & FanOnTime \\
\hline & & Desired Fan & FanOffTime \\
\hline & Desired & & FanSpeed \\
\hline & Properties & & LamponTime \\
\hline & & Desired Lamp & LampOffTime \\
\hline & & & Brightness \\
\hline & & Recired Pumn & PumpTime \\
\hline & & Desileu rump & PumpPeriod \\
\hline Properties & & Reported Interval & \\
\hline & & & FanOnTime \\
\hline & & Reported Fan & FanOffTime \\
\hline & & & FanSpeed \\
\hline & Reported & & LampOnTime \\
\hline & Properties & Reported Lamp & LampOffTime \\
\hline & & & Brightness \\
\hline & & Renorted Pumn & PumpTime \\
\hline & & Reported rump & PumpPeriod \\
\hline & & Reboot Number & \\
\hline
\end{tabular}

Figure 4.4: Plant wall system property object.

and light actuators. The desired/reported interval property is to adjust the time period that Edison reports sensor values to the cloud. The reboot num- 
ber property in the reported property is used to check if a reboot has been completed.

The details of this solution implementation is presented in Paper III.

\subsection{Visualization}

Visualization plays an essential role in understanding the patterns and relationships between different time series variables. In our framework, a web-based visualization application is developed to better analyze and find the underlying stories behind the data, and simplify the interaction with the actuators. Three commonly used visual representations within the data visualization community, namely Line graph, Stacked Area graph and Horizon graph, are implemented to ensure the user have a variety of representation methods to analyze the data.

In the Azure platform, a web application service is created to enable the visualization application while a container registry service is required to host the docker image repository so that the web application can be upgraded by pushing a new docker image to the container registry.

The presented application is divided into two focus areas: live stream data analysis and historical data analysis. With this setup the user can simply focus on current status of each IoT device or analyze historical data to better understand patterns.

- Live stream With this view the user is able to keep tracking and analyzing individual device in a live manner. This view consists of two parts, a control part and a live stream part. In the control part the user is able to directly manipulate the device by changing the device twin properties or invoking direct methods. The changes will be synchronized to the IoT Hub by calling Azure IoT Hub service SDK in the backend, and then applied to the device. The live stream part, which comprises several line graphs, is used for tracking the current status of the monitored environment. The application always listens to the IoT Hub event endpoint. Whenever a message is published in the endpoint, the application determines to visualize it or not depending on the currently selected device ID.

- Historical The second part of the application is focusing on the historical data. This view consists of three parts to analyze the data with; small multiples of Line graphs, a Stacked Area graph, and a Horizon graph. With this view, the user is able to analyze the historical data collected in order to have a overview of the long-term device status or environment fluctuations and to discover patterns.

For details of the web-based visualization interface, please refer to Paper IV. 


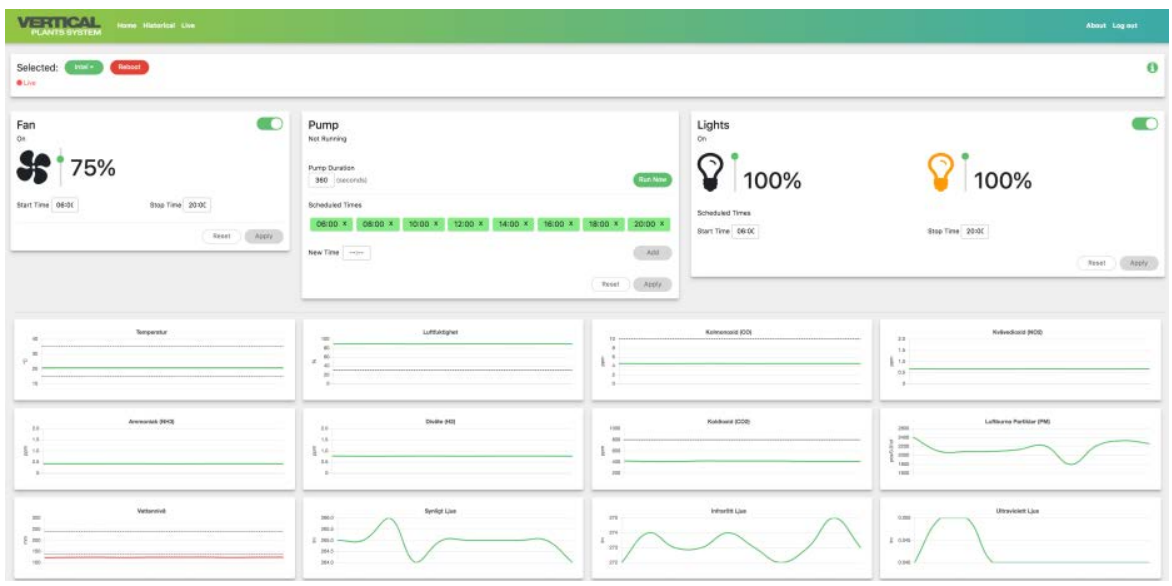

(a) Live stream and control functions

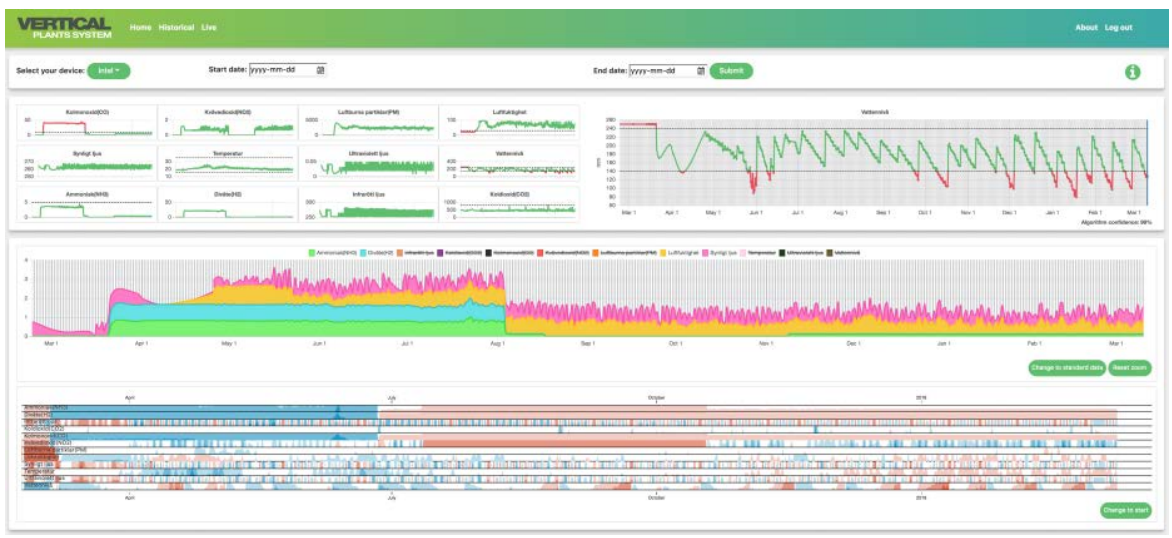

(b) Historical data

Figure 4.5: Web-based visualization application

\subsection{Intelligence}

With the prevalent application of machine learning and data mining, harvesting intelligence from the massive data becomes a promising goal of IoT. One popular intelligent application is predictive maintenance (PdM) that has been found adoption in various industries. PdM guarantees the quality of service of a system by having a comprehensive monitoring of historical and current conditions and providing prediction of upcoming failures so as to enable maintenance activities to react in advance.

In the context of our plant wall system, one promising task is to utilize collected environmental data to realize predictive maintenance of the indoor 


\begin{tabular}{|c|c|c|c|c|c|c|c|c|c|c|c|}
\hline Model & $\begin{array}{l}\text { Hidden } \\
\text { Lay- } \\
\text { ers }\end{array}$ & Neurons & $\begin{array}{l}\text { Total } \\
\text { Params }\end{array}$ & $\begin{array}{l}\text { Training } \\
\text { Time }\end{array}$ & $\begin{array}{l}\text { MAE } \\
\left(D_{\text {test }}\right)\end{array}$ & $\begin{array}{l}\text { MSE } \\
\left(D_{\text {test }}\right)\end{array}$ & $\begin{array}{l}\text { MAPE } \\
\left(D_{\text {test }}\right)\end{array}$ & AUC & $\begin{array}{l}\text { Opt. } \\
\text { THOLD } \\
\text { ( x Std) }\end{array}$ & $\begin{array}{l}\text { Opt. } \\
\text { TPR(\%) }\end{array}$ & $\begin{array}{l}\text { Opt. } \\
\text { FPR(\%) }\end{array}$ \\
\hline $\begin{array}{l}\text { Regression } \\
\text { (pred) }\end{array}$ & I & I & I & $1.8 \mathrm{~s}$ & 4.73 & 42.08 & 0.01 & 0.9912 & 0.9 & 98.8 & 4.8 \\
\hline ANN (pred) & 3 & $\begin{array}{l}{[200,} \\
200,50]\end{array}$ & 52501 & $60 \mathrm{~s}$ & 5.6 & 56.4 & 0.01 & 0.9897 & 1.0 & 98.3 & 4.7 \\
\hline $\begin{array}{l}\text { LSTM } \\
\text { (pred) }\end{array}$ & 1 & [128] & 66689 & $230 \mathrm{~s}$ & 5.3 & 51.4 & 0.01 & 0.944 & 0.81 & 98.9 & 4.9 \\
\hline $\begin{array}{l}\text { bi-LSTM } \\
\text { (pred) }\end{array}$ & 1 & [256] & 133377 & $380 \mathrm{~s}$ & 7.5 & 82.4 & 0.02 & 0.9964 & 1.31 & 98.4 & 2.4 \\
\hline $\begin{array}{l}\text { ANN with } \\
\text { temporal } \\
\text { features } \\
\text { (pred) }\end{array}$ & 3 & $\begin{array}{l}{[200,} \\
200,50]\end{array}$ & 64701 & $60 \mathrm{~s}$ & 6.8 & 73.9 & 0.02 & 0.9950 & 1.4 & 97.1 & 3.2 \\
\hline $\mathrm{AE}(\mathrm{sw})$ & 3 & $\begin{array}{l}{[128,} \\
32,128]\end{array}$ & 11050 & $10 \mathrm{~s}$ & 3.1 & 16.8 & 0.007 & 0.9981 & 0.1725 & 99.0 & 0.7 \\
\hline LSTM (sw) & 1 & [128] & 66689 & $30 \mathrm{~s}$ & 2.6 & 14.5 & 0.006 & 0.9964 & 0.105 & 98.8 & 3.1 \\
\hline $\begin{array}{l}\text { bi-LSTM } \\
\text { (sw) }\end{array}$ & 1 & [256] & 133377 & $40 \mathrm{~s}$ & 4.6 & 38.8 & 0.01 & 0.9978 & 0.16 & 99.4 & 6.7 \\
\hline $\begin{array}{l}\text { LSTM } \\
\text { multi-layer } \\
\text { (sw) }\end{array}$ & 5 & $\begin{array}{l}{[128,} \\
64, \quad 32, \\
64,128]\end{array}$ & 252161 & $90 \mathrm{~s}$ & 5.9 & 70.1 & 0.01 & 0.9928 & 0.26 & 98.3 & 5.0 \\
\hline $\begin{array}{l}\text { LSTM-ED } \\
(\mathrm{sw})\end{array}$ & 2 & $\begin{array}{l}{[128,} \\
128]\end{array}$ & 198273 & $50 \mathrm{~s}$ & 4.7 & 44.6 & 0.01 & 0.9967 & 0.25 & 98.8 & 1.8 \\
\hline $\begin{array}{l}\text { LSTM-ED } \\
\text { multi-layer } \\
\text { (sw) }\end{array}$ & 4 & $\begin{array}{l}{[128,} \\
64, \quad 64, \\
128]\end{array}$ & 247937 & $80 \mathrm{~s}$ & 5.7 & 75.2 & 0.01 & 0.9957 & 0.26 & 99.0 & 4.4 \\
\hline $\begin{array}{l}\mathrm{AE} \\
\text { (overlapped } \\
\text { sw) }\end{array}$ & 3 & $\begin{array}{l}{[128,} \\
32,128]\end{array}$ & 11050 & $40 \mathrm{~s}$ & 0.02 & 0.001 & 5.1 & 0.8392 & 0.0005 & 86.6 & 55.3 \\
\hline $\begin{array}{l}\text { LSTM (over- } \\
\text { lapped sw) }\end{array}$ & 1 & [128] & 66560 & $270 \mathrm{~s}$ & 0.55 & 0.47 & 0.001 & 0.8296 & 0.016 & 71.8 & 16.5 \\
\hline SVM & 1 & 1 & 1 & 1 & 1 & 1 & 1 & 0.96 & 1 & 86.6 & 9.6 \\
\hline Isolate forest & I & 1 & 1 & 1 & 1 & I & 1 & 0.9525 & 1 & 90.5 & 7.3 \\
\hline PCA & 1 & 1 & 1 & 1 & 1 & 1 & 1 & 0.6845 & 1 & 78.6 & 48.5 \\
\hline
\end{tabular}

Figure 4.6: Point anomaly detection comparison.

climate with a vertical plant wall. The indoor climate data stream is continuously processed and analyzed by the cloud server in a near real-time manner to detect potential anomalies of environmental parameters. When anomalies are detected, alarms can be automatically triggered and sent to the administrator and reactive measures can be taken. We categorize anomalies into point anomalies and contextual anomalies. Point anomalies is a single outlier value that can be caused by software errors, switch noise in the circuit or temporary interference of sensor readings. Contextual anomalies refer to a series of data points that are inappropriate in a given period of time, although individually, the data values are within the normal range of an environmental parameter. Point anomalies might not be harmful to the environment but can bring bias to further data processing and analysis in later steps. Contextual anomalies indicate external interference to an indoor climate, thus, need to be detected as early as possible.

We adopt several neural network-based anomaly detection methods into the solution, e.g., artificial neural network (ANN), recurrent neural network (RNN), autoencoder (AE), long short-term memory neural network (LSTM), etc. We benchmark the performance of different methods in terms of detecting both point anomalies and contextual anomalies. In addition, some baseline models that have been widely utilized are also brought into the benchmark as a reference. In the benchmarking, both prediction-based and pattern recognition-based detection techniques are compared. Figure 4.6 shows the 


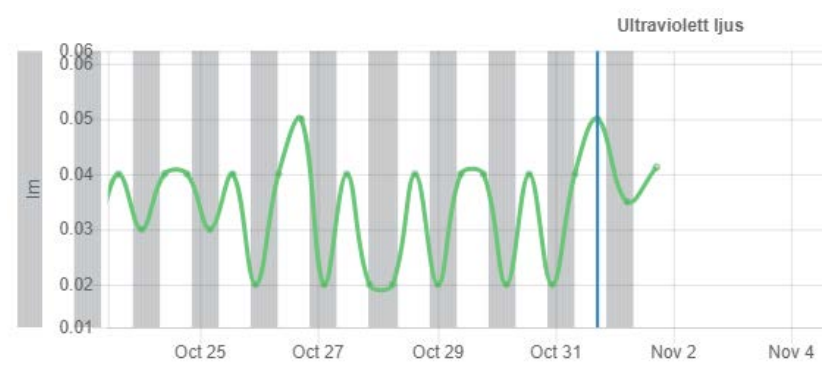

Figure 4.7: Prediction Example.

results of performance comparison of different methods in point anomaly detection. The higher value in the AUC column indicates better performance. The results suggest that, in general pattern recognition-based detection techniques have superior performance than prediction-based ones. Among all detection algorithms, an autoencoder neural network ourperforms the others in terms of network structure, training difficulty, and point anomaly detection performance.

Finally, as a proof-of-concept, a regression-based method is deployed to the web-application in the container, to have real-time prediction of the sensor data. Figure 4.7 depicts an example of predicting ultraviolet light values. Once the new incoming data vary from the prediction to a large degree, alert can be generated.

The detailed benchmarking setup and results are present in Paper V. 



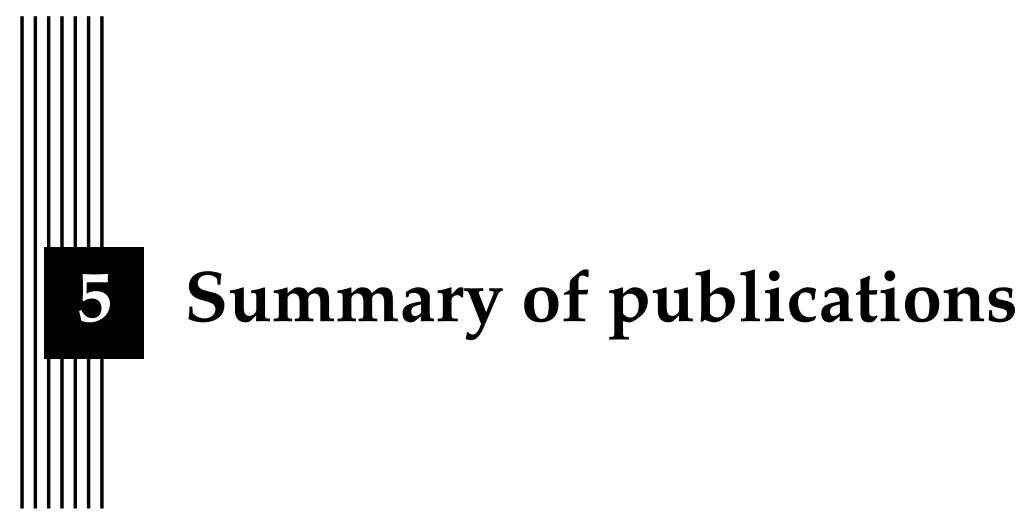

This chapter summaries the papers that are associated with this thesis.

Paper I: Y. Liu, K. Akram Hassan, M. Karlsson, Z. Pang and S. Gong, "A Data-Centric Internet of Things Framework Based on Azure Cloud," in IEEE Access, vol. 7, pp. 53839-53858, 2019.

Summary: In this study, a data-centric IoT framework based on Azure cloud is proposed to tackle the interoperability, co-existence, device management and data consistency challenges in the IoT practice. The system architecture is provided and the implementation details are described as a reference to IoT practioners.

Contribution: Proposed the data-centric IoT framework, and realized the implementation as a proof of concept.

Paper II: Y. Liu, Z. Pang, G. Dán, D. Lan and S. Gong, "A Taxonomy for the Security Assessment of IP-Based Building Automation Systems: The Case of Thread," in IEEE Transactions on Industrial Informatics, vol. 14, no. 9, pp. 4113-4123, Sept. 2018.

Summary: In this study, a security assessment taxonomy for building automation networks is proposed with consideration of the whole life cycle of a network device. The taxonomy is then applied to the security analysis of Thread protocol. The results show that Thread has superior security performance than its competitors. Several security enhancement measures are also proposed to strengthen the security level of Thread. 
Contribution: Proposed the security assessment taxonomy, performed the security analysis of Thread protocol and proposed countermeasures to enhance Thread security.

Paper III: Y. Liu, K. Akram Hassan, M. Karlsson, O. Weister and S. Gong, "Active Plant Wall for Green Indoor Climate Based on Cloud and Internet of Things," in IEEE Access, vol. 6, pp. 33631-33644, 2018.

Summary: In this study, a remote monitoring and management solution for green plant wall industry based on the Azure cloud platform is proposed, implemented and deployed in real practice. Compared to other solutions, our solution features in bi-directional communications, native-IP support, easy deployment, reliability and scalability, and low cost.

Contribution: Proposed and implemented the Azure-based remote monitoring and management solution for green plant walls.

Paper IV: K. Akram Hassan,Y. Liu, L. Besançon, J. Johansson, and N. Rönnberg, "A Study on Visual Representations for Active Plantwall Data Analysis," in Data 4.2, 2019.

Summary: This study presents a user study on the representation of indoor climate data. Three visualization methods, namely Line graph, Horizon graph and Stacked area graph, are explored. The results show that the Line graph is preferred for providing overview, the Horizon graph for detailed analysis, revealing patterns and showing discernible trends while the Stacked area graph was generally not preferred. This study facilities further researches on the analysis of multivariate temporal data.

Contribution: Implemented the testbed for collecting indoor climate data and involved in the analysis of different visualization methods.

Paper V: Y. Liu, M. Karlsson, Z. Pang and S. Gong, "Towards Predictive Maintenance of Indoor Climate with A Vertical Plant Wall and Neural Networks," Manuscript, 2019.

Summary: In this paper, prediction-based and pattern recognition-based anomaly detection methods are reviewed and benchmarked with respect to point anomalies and contextual anomalies using real indoor climate data. The results show that a neuron network-based anomaly detection method outperforms the traditional models like SVM, PCA and isolate forest. A regression-based method is also deployed into the cloud-based green plant wall system as a validation to realize predictive maintenance for indoor climate.

Contribution: Benchmarked different prediction-based and pattern recognition-based anomaly detection methods, and deployed regressionbased methods into real plant wall system to achieve predictive maintenance. 


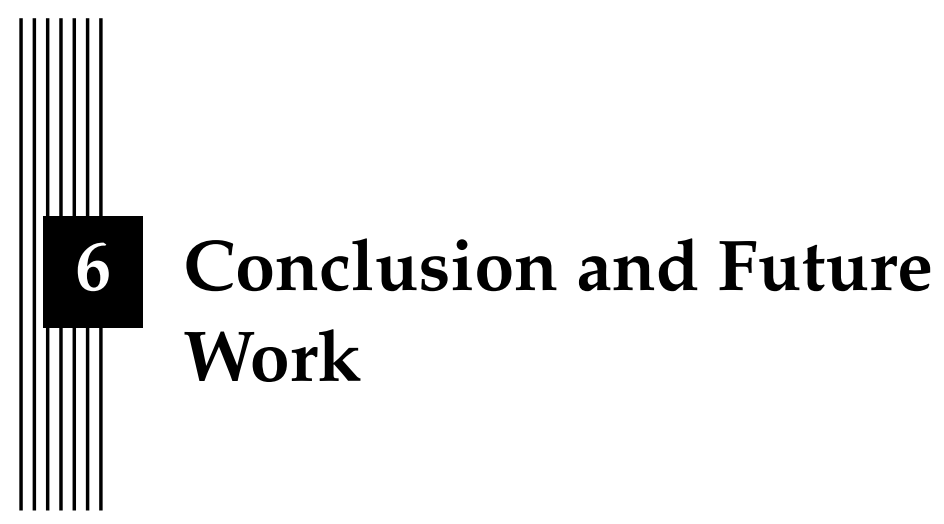

With the evolution of technology, new communication standards are emerging all the way, catering to the tendency that traditional domainspecific M2M communications are migrating towards the broader IoT paradigm which envisions a world fully connected by the Internet infrastructure in the near future. IoT has been reshaping our society in various aspects, from the way in which we are living to industrial production. With the of emergence and popularity of cloud computing, the integration of IoT with cloud platform is in the ascendant, which facilitates the deployment of IoT solutions and applications to a large extent, thanks to the rapidly growing power in computing and processing, the flexible data storage, and the deep learning and data mining-based intelligent services. During this IoT revolution, a series of challenges are identified and cannot be resolved by the cloud suppliers as they mainly focus on software and platform services but can hardly contribute to the end devices due to the heterogeneity of hardware and communication protocols. Therefore, in this thesis, we proposed a data-centric IoT framework based on public cloud platform to tackle these challenges, i.e., the interoperability and compatibility among link-layer protocols, the uniform device management scheme, and the heterogeneity of massive data from diverse IoT applications. This framework integrates three promising protocols, i.e., WiFi, Thread and LoRaWAN, to satisfy different needs for local, personal and wide area networks in practice. Azure cloud platform is adopted to simplify the device management and data storage, benefiting from the uniform device model and data model. We also exem- 
plify the framework with a case study that is to develop a remote monitoring and management system for green plant wall industry to ease the maintenance and plantcare costs. Furthermore, three novel visualization methods are proposed to better reflect the environmental data, and a neuron networkbased anomaly detection method is employed in the system to realize predictive maintenance of the indoor climate, which suggests the proposed framework is fully functional and able to support the deployment of solutions for massive IoT and broadband IoT from communication to computing and finally towards intelligence.

As the requirement on latency is much stringent for critical IoT applications and industrial automation IoT use cases, recently, edge computing has become an emerging paradigm and accelerates the development of critical IoT and industrial IoT. In the future, more efforts will be spent on integrating edge computing into the existing framework. Specifically, the benchmarking of resource consumption for different type of tasks and the partitioning of communication, computing and intelligence services, are worthy of further investigation. 


\section{Bibliography}

[1] P.P. Ray. "A survey on Internet of Things architectures". In: Journal of King Saud University - Computer and Information Sciences 30.3 (2018), pp. 291-319. ISSN: 1319-1578. DOI: https : / / doi .org/10.1016/ j.jksuci.2016.10.003.

[2] Jan Höller, Vlasios Tsiatsis, Catherine Mulligan, Stamatis Karnouskos, Stefan Avesand, and David Boyle. "Chapter 2 - M2M to IoT - The Vision". In: From Machine-To-Machine to the Internet of Things. Ed. by Jan Höller, Vlasios Tsiatsis, Catherine Mulligan, Stamatis Karnouskos, Stefan Avesand, and David Boyle. Oxford: Academic Press, 2014, pp. 937. ISBN: 978-0-12-407684-6. DOI: https : / / doi . org / 10 . 1016 / B 978-0-12-407684-6.00002-4.

[3] Cellular IoT Evolution for Industry Digitalization. White Paper. Ericsson, Jaunary 2019.

[4] E. Sisinni, A. Saifullah, S. Han, U. Jennehag, and M. Gidlund. "Industrial Internet of Things: Challenges, Opportunities, and Directions". In: IEEE Transactions on Industrial Informatics 14.11 (Nov. 2018), pp. 47244734. ISSN: 1551-3203. DOI: 10.1109 /TII.2018.2852491.

[5] A. Zanella, N. Bui, A. Castellani, L. Vangelista, and M. Zorzi. "Internet of Things for Smart Cities". In: IEEE Internet of Things Journal 1.1 (Feb. 2014), pp. 22-32. ISSN: 2327-4662. DOI: 10 . 1109 / JIOT . 2014 . 2306328.

[6] Massive IoT in the City. Report. Ericsson, Nov. 2016. 
[7] M. E. Johansson Zhibo Pang Yuxin Cheng and G. Bag. "Work-inprogress: Industry-friendly and native-IP wireless communications for building automation". In: 2015 1st International Conference on Industrial Networks and Intelligent Systems (INISCom). Mar. 2015, pp. 163-167. DOI: 10.4108 /icst. iniscom.2015.258563.

[8] J. Peng, X. Zhang, Z. Lei, B. Zhang, W. Zhang, and Q. Li. "Comparison of Several Cloud Computing Platforms". In: 2009 Second International Symposium on Information Science and Engineering. Dec. 2009, pp. 23-27. DOI: $10.1109 /$ ISISE.2009.94.

[9] Saurabh Singh, Young-Sik Jeong, and Jong Hyuk Park. "A survey on cloud computing security: Issues, threats, and solutions". In: Journal of Network and Computer Applications 75 (2016), pp. 200-222. ISSN: 10848045. DOI: https://doi.org/10.1016/j.jnca.2016.09.002.

[10] WiFi Alliance. Next generation Wi-Fi: The future of connectivity. Dec. 2018. URL: https: / /www.wi-fi.org.

[11] Thread Specification 1.1.1. Specification. Thread Group, Feb. 2017.

[12] D. Lan, Z. Pang, C. Fischione, Y. Liu, A. Taherkordi, and F. Eliassen. "Latency Analysis of Wireless Networks for Proximity Services in Smart Home and Building Automation: The Case of Thread". In: IEEE Access 7 (2019), pp. 4856-4867. ISSN: 2169-3536.

[13] E. Azoidou, Z. Pang, Y. Liu, D. Lan, G. Bag, and S. Gong. "Battery Lifetime Modeling and Validation of Wireless Building Automation Devices in Thread". In: IEEE Transactions on Industrial Informatics 14.7 (July 2018), pp. 2869-2880. ISSN: 1551-3203.

[14] Benchmarking Bluetooth Mesh, Thread, and Zigbee Network Performance. Webinar. Silicon Labs, June 2018.

[15] LoRaWAN 1.0.3 Specification. Specification. LoRa Alliance, July 2018.

[16] Machine eXchange Protocol. White Paper. MXC Foundation, May 2018.

[17] Microsoft. Microsoft Azure IoT reference architecture. Sept. 2018. URL: https : / / azure. microsoft. com/en-us / blog/azure-iotreference-architecture-2-1-release/.

[18] C. Gomez and J. Paradells. "Wireless home automation networks: A survey of architectures and technologies". In: IEEE Communications Magazine 48.6 (June 2010), pp. 92-101. ISSN: 0163-6804. DOI: 10 . 1109 / MCOM. 2010.5473869.

[19] W. Granzer, F. Praus, and W. Kastner. "Security in Building Automation Systems". In: IEEE Transactions on Industrial Electronics 57.11 (Nov. 2010), pp. 3622-3630. ISSN: 0278-0046. DOI: 10 . 1109 / TIE . 2009. 2036033. 
[20] Wolfgang Granzer and Wolfgang Kastner. "Security Analysis of Open Building Automation Systems". In: Proc. of SAFECOMP. 2010, pp. 303316.

[21] T Goodspeed. "Extracting Keys from Second Generation Zigbee Chips". In: Black Hat USA Conference. 2009.

[22] Y. Liu, Z. Pang, G. Dán, D. Lan, and S. Gong. "A Taxonomy for the Security Assessment of IP-Based Building Automation Systems: The Case of Thread". In: IEEE Transactions on Industrial Informatics 14.9 (Sept. 2018), pp. 4113-4123. ISSN: 1551-3203. DOI: 10 . 1109 / TII . 2018.2844955.

[23] D. J. Fehér and B. Sandor. "Effects of the WPA2 KRACK Attack in Real Environment". In: 2018 IEEE 16th International Symposium on Intelligent Systems and Informatics (SISY). Sept. 2018, pp. 000239-000242.

[24] LoRaWAN Security. White paper. LoRa Alliance, Feb. 2017.

[25] Microsoft. Azure IoT Hub Guide. 2019. URL: https : / / docs . microsoft.com/en-us/azure/iot-hub.

[26] Sue Murphy Julie Francis Gail Hall and John Rayner. Growing Green Guide. Feb. 2014. URL: http: / /www. growinggreenguide.org/.

[27] Y. Liu, K. Akram Hassan, M. Karlsson, Z. Pang, and S. Gong. "A DataCentric Internet of Things Framework Based on Azure Cloud". In: IEEE Access 7 (2019), pp. 53839-53858. ISSN: 2169-3536. DOI: 10.1109 / ACCESS.2019.2913224.

[28] Y. Liu, K. Akram Hassan, M. Karlsson, O. Weister, and S. Gong. “Active Plant Wall for Green Indoor Climate Based on Cloud and Internet of Things". In: IEEE Access 6 (2018), pp. 33631-33644. ISSN: 2169-3536. DOI: $10.1109 /$ ACCESS.2018.2847440.

[29] Kahin Akram Hassan, Yu Liu, Lonni Besançon, Jimmy Johansson, and Niklas Rönnberg. "A Study on Visual Representations for Active Plant Wall Data Analysis". In: Data 4.2 (2019). ISSN: 2306-5729. DOI: 10 . 3390 / data4020074. URL: https : / / www . mdpi . com/2306$5729 / 4 / 2 / 74$.

[30] Y. Liu, M. Karlsson, Z. Pang, and S. Gong. “Towards Predictive Maintenance of Indoor Climate with A Vertical Plant Wall and Neural Networks". In: Manuscript (2019). 


\section{Papers}

The papers associated with this thesis have been removed for copyright reasons. For more details about these see:

http://urn.kb.se/resolve?urn=urn:nbn:se:liu:diva-159770 


\section{FACULTY OF SCIENCE AND ENGINEERING}

Linköping Studies in Science and Technology, Licentiate Thesis No. 1850, 2019 Department of Science and Technology

Linköping University

SE-60174 Norrköping, Sweden

www.liu.se

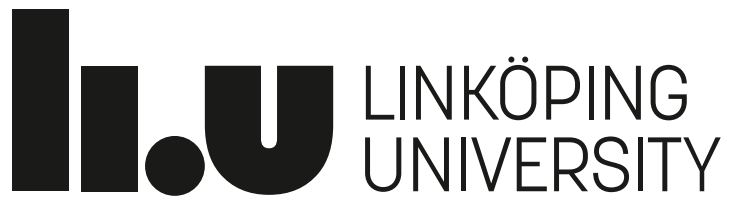

